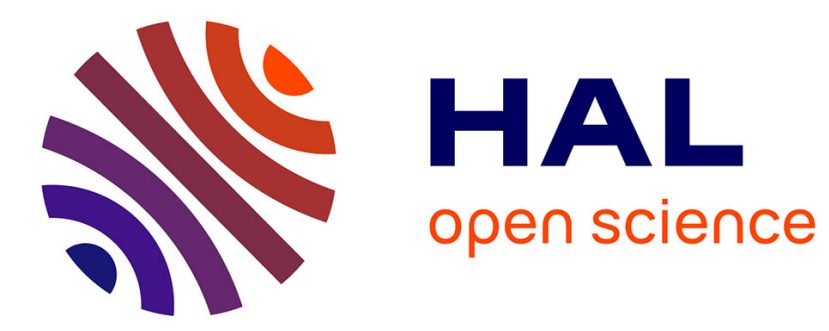

\title{
Smooth projective symmetric varieties with Picard number one
}

Alessandro Ruzzi

\section{To cite this version:}

Alessandro Ruzzi. Smooth projective symmetric varieties with Picard number one. International Journal of Mathematics, 2011, 22 (2), pp.145-177. 10.1142/S0129167X11005678 . hal-00578661

\section{HAL Id: hal-00578661 \\ https://hal.science/hal-00578661}

Submitted on 21 Mar 2011

HAL is a multi-disciplinary open access archive for the deposit and dissemination of scientific research documents, whether they are published or not. The documents may come from teaching and research institutions in France or abroad, or from public or private research centers.
L'archive ouverte pluridisciplinaire HAL, est destinée au dépôt et à la diffusion de documents scientifiques de niveau recherche, publiés ou non, émanant des établissements d'enseignement et de recherche français ou étrangers, des laboratoires publics ou privés. 


\title{
SMOOTH PROJECTIVE SYMMETRIC VARIETIES WITH PICARD NUMBER ONE
}

\author{
ALESSANDRO RUZZI
}

\begin{abstract}
We classify the smooth projective symmetric $G$-varieties with $\mathrm{Pi}$ card number one (and $G$ semisimple). Moreover we prove a criterion for the smoothness of the simple (normal) symmetric varieties whose closed orbit is complete. In particular we prove that, given a such variety $X$ which is not exceptional, then $X$ is smooth if and only if an appropriate toric variety contained in $X$ is smooth.
\end{abstract}

A Gorenstein normal algebraic variety $X$ over $\mathbb{C}$ is called a Fano variety if the anticanonical divisor is ample. The Fano surfaces are classically called Del Pezzo surfaces. The importance of Fano varieties in the theory of higher dimensional varieties is similar to the importance of Del Pezzo surfaces in the theory of surfaces. Moreover Mori's program predicts that every uniruled variety is birational to a fiberspace whose general fiber is a Fano variety (with terminal singularities).

Often it is useful to subdivide the Fano varieties in two kinds: the Fano varieties with Picard number one and the Fano varieties with Picard number strictly greater than one. For example, there are many results which give an explicit bound to some numerical invariants of a Fano variety (depending on the Picard number and on the dimension of the variety). Often there is an explicit expression for the Fano varieties of Picard number one and another expression for the remaining Fano varieties.

We are mainly interested in the smooth projective spherical varieties with Picard number one. The smooth toric (resp. homogeneous) projective varieties with Picard number one are just projective spaces (resp. G/P with G simple and $\mathrm{P}$ maximal parabolic). We classify the smooth projective symmetric $G$-varieties whose Picard group is isomorphic to $\mathbb{Z}$ (with the hypothesis $G$ semisimple). One can easily show that they are all Fano, because the canonical bundle cannot be ample.

In [14] there are some partial results regarding the classification of smooth Fano projective symmetric varieties with with low rank (and Picard number greater than one). In [13], we give a geometrical description of these varieties; in particular, we determine their group of automorphism. Moreover, if the action of this last group is not transitive, then the variety is a linear section of a generalized flag variety and it is lied to exceptional groups. See also [11] for a classification of the smooth projective horospherical varieties with Picard number one.

Let $G$ be a connected reductive algebraic group over $\mathbb{C}$ and let $\theta$ be an involution of $G$. Let $H$ be a closed subgroup of $G$ such that $G^{\theta} \subset H \subset N_{G}\left(G^{\theta}\right)$. We will say that a normal equivariant embedding of $G / H$ is a symmetric variety. Any symmetric variety can be covered by open $G$-subvarieties which are simple, i.e. have one closed $G$-subvariety. In Section 1 we recall some known facts about symmetric varieties and we fix the notations. In Section 2 we give a criterion for the smoothness of the simple symmetric varieties whose closed orbit is complete (see Theorem 2.1 and Theorem 2.2). It is easily showed that every complete symmetric variety is 
covered by simple open symmetric $G$-subvarieties whose closed orbits are complete. We generalize the results of Timashev (see [16]) and Renner (see [12]) on the equivariant embeddings of reductive groups. The idea of the proof is the following one. First we reduce to the affine case, though there are some technical problems when the variety is exceptional (see [5] for a definition of exceptional symmetric variety). Given an affine $X$, we define a toric variety $\overline{T \cdot x_{0}}$ contained in $X$ and we prove that $X$ is smooth if and only $\overline{T \cdot x_{0}}$ is smooth. In such case we relate the combinatorial description of $\overline{T \cdot x_{0}}$ to the colored fan of $X$ (see [3] for a definition of colored fan).

In Section 3 we use the previous criterion to classify the smooth complete symmetric $G$-varieties with Picard number one and $G$ semisimple (see Theorem 3.1). We do not classify directly the projective smooth symmetric varieties with Picard number one because there is a combinatorial classification of the complete symmetric varieties, but it is not so easy to say which ones are projective. We prove that the smooth complete symmetric varieties with Picard number one (and $G$ semisimple) have at most two closed orbits. Because of this fact we easily prove that they are all projective. Observe that all the simple complete symmetric varieties are projective by a theorem of Sumihiro.

\section{NOTATIONS AND BACKGROUND}

In this section we introduce the necessary notations. The reader interested to the embedding theory of spherical varieties can see [3] or [17]. In [18] is explained such theory in the particular case of the symmetric varieties. Let $G$ be a connected reductive algebraic group over $\mathbb{C}$ and let $\theta$ be an involution of $G$. Let $H$ be a closed subgroup of $G$ such that $G^{\theta} \subset H \subset N_{G}\left(G^{\theta}\right)$. We say that $G / H$ is a symmetric homogeneous variety. An equivariant embedding of $G / H$ is the data of a $G$-variety $X$ together with an equivariant open immersion $G / H \hookrightarrow X$. A normal $G$-variety is called a spherical variety if it contains a dense orbit under the action of an arbitrarily chosen Borel subgroup of $G$. One can show that an equivariant embedding of $G / H$ is a spherical variety if and only if it is normal (see [5] Proposition 1.3). In this case we say that it is a symmetric variety. We say that a subtorus of $G$ is split if $\theta(t)=t^{-1}$ for all its elements $t$. We say that a split torus of $G$ of maximal dimension is a maximal split torus and that a maximal torus containing a maximal split torus is maximally split. One can prove that any maximally split torus is $\theta$ stable (see [17] Lemma 26.5). We fix arbitrarily a maximal split torus $T^{1}$ and a maximally split torus $T$ containing $T^{1}$. Let $R_{G}$ be the root system of $G$ with respect to $T$ and let $R_{G}^{0}$ be the subroot system of the roots fixed by $\theta$. We define $R_{G}^{1}=R_{G} \backslash R_{G}^{0}$. We can choose a Borel subgroup $B$ containing $T$ such that, if $\alpha$ is a positive root in $R_{G}^{1}$, then $\theta(\alpha)$ is negative (see [5] Lemma 1.2). One can prove that $B H$ is dense in $G$ (see [5] Proposition 1.3). Let $D(G / H)$ be the set of $B$-stable prime divisors of $G / H$; its elements are called colors. Since $B H / H$ is an affine open orbit, the colors are the irreducible components of $(G / H) \backslash(B H / H)$. We say that a spherical variety is simple if it contains one closed orbit. Let $X$ be a simple symmetric variety with closed orbit $Y$. Let $D(X)$ be the subset of $D(G / H)$ consisting of the colors whose closure in $X$ contains $Y$. We say that $D(X)$ is the set of colors of $X$.

To each prime divisor $D$ of $X$, we can associate the normalized discrete valuation $v_{D}$ of $\mathbb{C}(G / H)$ whose ring is the local ring $\mathcal{O}_{X, D}$. One can prove that $D$ is $G$-stable if and only if $v_{D}$ is $G$-invariant, i.e. $v_{D}(s \cdot f)=v_{D}(f)$ for each $s \in G$ and $f \in \mathbb{C}(G / H)$. Let $N$ be the set of all $G$-invariant valuations of $\mathbb{C}(G / H)$ taking value in $\mathbb{Z}$ and let $N(X)$ be the set of the valuations associated to the $G$-stable prime divisors of $X$. Observe that each irreducible component of $X \backslash(G / H)$ has codimension 1 because $G / H$ is affine. (To define $N(X)$ we do not need that $X$ is simple). Let 
$S:=T / T \cap H \simeq T \cdot x_{0}$, where $x_{0}=H / H$ denotes the base point of $G / H$. One can show that the group $\mathbb{C}(G / H)^{(B)} / \mathbb{C}^{*}$ is isomorphic to the character group $\chi(S)$ of $S$ (see [18] §2.3); in particular, it is a free abelian group. We define the rank of $G / H$ as the rank of $\chi(S)$; it is also equal to the dimension of $S$. We can identify the dual group $\operatorname{Hom}_{\mathbb{Z}}\left(\mathbb{C}(G / H)^{(B)} / \mathbb{C}^{*}, \mathbb{Z}\right)$ with the group $\chi_{*}(S)$ of one-parameter subgroups of $S$; so we can identify $\chi_{*}(S) \otimes \mathbb{R}$ with $H_{0} m_{\mathbb{Z}}(\chi(S), \mathbb{R})$. The restriction map to $\mathbb{C}(G / H)^{(B)} / \mathbb{C}^{*}$ is injective over $N$ (see [3] $\S 3.1$ Corollaire 3 ), so we can identify $N$ with a subset of $\chi_{*}(S) \otimes \mathbb{R}$. We say that $N$ is the valuation semigroup of $G / H$. Indeed, $N$ is the semigroup constituted by the vectors contained in the intersection of the lattice $\chi_{*}(S)$ with an appropriate rational polyhedral convex cone $\mathcal{C} N$; we say that $\mathcal{C} N$ is the valuation cone. For each color $D$, we define $\rho(D)$ as the restriction of $v_{D}$ to $\chi(S)$. In general, the map $\rho: D(G / H) \rightarrow \chi_{*}(S) \otimes \mathbb{R}$ is not injective.

Let $C(X)$ be the cone in $\chi_{*}(S) \otimes \mathbb{R}$ generated by $N(X)$ and $\rho(D(X))$. We can recover $N(X)$ from $C(X)$. Indeed $N(X)$ consists of the primitive vectors of the onedimensional faces of $C(X)$ which intersect $N$ (we say that a vector $v \in \chi_{*}(S) \backslash\{0\}$ is primitive if an equality $v=a v^{\prime}$, where $v^{\prime}$ is a vector in $\chi_{*}(S)$ and $a$ is a positive integer, implies $a=1)$. We denote by cone $\left(v_{1}, \ldots, v_{r}\right)$ the cone generated by the vectors $v_{1}, \ldots, v_{r}$. Given a cone $C$ in $\chi_{*}(S) \otimes \mathbb{R}$ and a subset $D$ of $D(G / H)$, we say that $(C, D)$ is a colored cone if:

(1) $C$ is generated by $\rho(D)$ and by a finite number of vectors in $N$;

(2) the relative interior of $C$ intersects $\mathcal{C} N$.

Proposition 1.1 (see [3] $§ 3.3$ Théorème). The map $X \rightarrow(C(X), D(X))$ is a bijection from the set of simple symmetric varieties to the set of colored cones.

Given a symmetric variety $\widetilde{X}$ (not necessarily simple), let $\left\{Y_{i}\right\}_{i \in I}$ be the set of $G$-orbits. Observe that $\tilde{X}$ contains a finite number of $G$-orbits, thus $\widetilde{X}_{i}:=\{x \in$ $\left.\widetilde{X} \mid \overline{G \cdot x} \supset Y_{i}\right\}$ is open in $\widetilde{X}$ and is a simple symmetric variety whose closed orbit is $Y_{i}$. We define $D(\widetilde{X})$ as the set $\bigcup_{i \in I} D\left(\widetilde{X}_{i}\right)$. The family $\left\{\left(C\left(\widetilde{X}_{i}\right), D\left(\widetilde{X}_{i}\right)\right)\right\}_{i \in I}$ is called the colored fan of $\tilde{X}$ and determines completely $\tilde{X}$. Moreover $\tilde{X}$ is complete if and only if $\mathcal{C} N \subset \bigcup_{i \in I} C\left(\widetilde{X}_{i}\right)$ (see [3] $\$ 3.4$ Théoremè 2).

Proposition 1.2 (see [3] $\S 3.4$ Théorème 1). A family $\left\{\left(C_{i}, D_{i}\right)\right\}_{i \in I}$ of colored cones is the colored fan of a symmetric variety if and only if

(1) given a colored cone $\left(C^{\prime}, D^{\prime}\right)$ such that $C^{\prime}$ is a face of $C_{i_{0}}$ and $D^{\prime}$ is the set $\left\{D \in D_{i_{0}} \mid \rho(D) \in C_{i_{0}}\right\}$ for an appropriate $i_{0} \in I$, then $\left(C^{\prime}, D^{\prime}\right)$ belongs to $\left\{\left(C_{i}, D_{i}\right)\right\}_{i \in I}$;

(2) the intersections of $\mathcal{C} N$ with the relative interiors of the $C_{i}$ are pairwise disjoint.

We recall the description of the sets $N$ and $\rho(D(G / H))$. Before that, we need to associate a root system to $G / H$. The subgroup $\chi(S)$ of $\chi\left(T^{1}\right)$ has finite index, so we can identify $\chi\left(T^{1}\right) \otimes \mathbb{R}$ with $\chi(S) \otimes \mathbb{R}$. Because $T$ is $\theta$-stable, $\theta$ induces an involution of $\chi(T) \otimes \mathbb{R}$ which we call again $\theta$. The inclusion $T^{1} \subset T$ induces an isomorphism of $\chi\left(T^{1}\right) \otimes \mathbb{R}$ with the -1 eigenspace of $\chi(T) \otimes \mathbb{R}$ under the action of $\theta$ (see [17] $\S 26$ ). Denoted by $W_{G}$ the Weyl group of $G$ (with respect to $T$ ), fix arbitrarily a $W_{G}$-invariant inner product $(\cdot, \cdot)$ over $\chi(T) \otimes \mathbb{R}$ which coincides with the product given by the Killing form over $\operatorname{span}_{\mathbb{R}}\left(R_{G}\right)$. We denote again $(\cdot, \cdot)$ the restriction of this inner product to $\chi\left(T^{1}\right) \otimes \mathbb{R}$. Thus we can identify $\chi\left(T^{1}\right) \otimes \mathbb{R}$ with its dual $\chi_{*}\left(T^{1}\right) \otimes \mathbb{R}$. The set $R_{G, \theta}:=\left\{\beta-\theta(\beta) \mid \beta \in R_{G}\right\} \backslash\{0\}$ is a root system in $\chi(S) \otimes \mathbb{R}$ (see [18] $\S 2.3$ Lemme), which we call the restricted root system of $(G, \theta)$; we call the non zero $\beta-\theta(\beta)$ the restricted roots. If $G$ is semisimple, then the rank of $G / H$ is equal to the rank of $R_{G, \theta}$, i.e. the dimension of $\operatorname{span}_{\mathbb{R}}\left(R_{G, \theta}\right)$. Usually we 
denote by $\beta$ (respectively by $\alpha$ ) a root of $R_{G}$ (respectively of $R_{G, \theta}$ ); often we denote by $\varpi$ (respectively by $\omega$ ) a weight of $R_{G}$ (respectively of $R_{G, \theta}$ ). In particular, we denote by $\varpi_{1}, \ldots, \varpi_{n}$ the fundamental weight of $R_{G}$ (we have chosen the basis of $R_{G}$ associated to $B$ ). Notice however that the weights of $R_{G, \theta}$ are weights of $R_{G}$. The involution $\iota:=-\varpi_{0} \cdot \theta$ of $\chi(T)$ preserves the set of simple roots; moreover $\iota$ coincides with $-\theta$ modulo the lattice generated by $R_{G}^{0}$ (see [17] p.169). Here $\varpi_{0}$ is the longest element of the Weyl group of $R_{G}^{0}$. We denote by $\alpha_{1}, \ldots, \alpha_{s}$ the elements of the basis $\left\{\beta-\theta(\beta) \mid \beta \in R_{G}\right.$ simple $\} \backslash\{0\}$ of $R_{G, \theta}$. Let $b_{i}$ be equal to $\frac{1}{2}$ if $2 \alpha_{i}$ belongs to $R_{G, \theta}$ and equal to 1 otherwise; for each $i$ we define $\alpha_{i}^{\vee}$ as the coroot $\frac{2 b_{i}}{\left(\alpha_{i}, \alpha_{i}\right)} \alpha_{i}$. The set $\left\{\alpha_{1}^{\vee}, \ldots, \alpha_{s}^{\vee}\right\}$ is a basis of the restricted dual root system $R_{G, \theta}^{\vee}$, namely the root system composed by the coroots of the restricted roots. We call the elements of $R_{G, \theta}^{\vee}$ the restricted coroots. Let $\omega_{1}, \ldots, \omega_{s}$ be the fundamental weights of $R_{G, \theta}$ with respect to $\left\{\alpha_{1}, \ldots, \alpha_{s}\right\}$ and let $\omega_{1}^{\vee}, \ldots, \omega_{s}^{\vee}$ be the fundamental weights of $R_{G, \theta}^{\vee}$ with respect to $\left\{\alpha_{1}^{\vee}, \ldots, \alpha_{s}^{\vee}\right\}$. Let $C^{+}$be the positive closed Weyl chamber of $\chi(S) \otimes \mathbb{R}$, i.e. the cone generated by the fundamental weights of $R_{G, \theta}$ and by the vectors orthogonal to $\operatorname{span}_{\mathbb{R}}\left(R_{G, \theta}\right)$. We call $-C^{+}$the negative Weyl chamber. Given a dominant weight $\lambda$ of $G$, we denote by $V(\lambda)$ the irreducible representation of highest weight $\lambda$.

If $G$ is semisimple and simply connected, then the lattice generated by the restricted roots is isomorphic to the character group of $T^{1} /\left(T^{1} \cap N_{G}\left(G^{\theta}\right)\right)$. Moreover the weight lattice of $R_{G, \theta}$ is $\{\varpi-\theta(\varpi) \mid \varpi \in \chi(T)\}$ and can be identified with the character group of $T^{1} / T^{1} \cap G^{\theta}$. We want to give another description of this lattice. We say that a dominant weight $\varpi \in \chi(T)$ is a spherical weight if $V(\varpi)$ contains a non-zero vector fixed by $G^{\theta}$ (in this definition we do not require that $G$ is semisimple). In this case, $V^{G^{\theta}}$ is 1 -dimensional and $\theta(\varpi)=-\varpi$. Thus we can think $\varpi$ as a vector in $\chi(S) \otimes \mathbb{R}$. One can show that the lattice generated by the spherical weights coincides with the weight lattice of $R_{G, \theta}$. See [4] Theorem 2.3 or [17] Proposition 26.4 for an explicit description of the spherical weights. Such description implies that the set of dominant weights of $R_{G, \theta}$ is the set of spherical weights and that $C^{+}$is the intersection of $\chi(S) \otimes \mathbb{R}$ with the positive closed Weyl chamber of the root system $R_{G}$. Sometimes we call $\sum \mathbb{Z}^{+} \omega_{i}^{\vee}$ the set of spherical coweights.

The set $N$ is equal to $-C^{+} \cap \chi_{*}(S)$; in particular it consists of the lattice vectors of the rational polyhedral convex cone $\mathcal{C} N=-C^{+}$. The set $\rho(D(G / H))$ is equal to $\left\{\alpha_{1}^{\vee}, \ldots, \alpha_{s}^{\vee}\right\}$ (see [18] $\S 2.4$ Propositions 1 and 2 ). Thus, given a simple symmetric variety $X$, the set $\rho(D(X))$ consists of the primitive generators of the 1-dimensional faces of $C(X)$ not contained in $-C^{+}$, because $\left(\alpha_{i}, \alpha_{j}^{\vee}\right)>0$ if and only if $i=j$. For each $i$ the fibre $\rho^{-1}\left(\alpha_{i}^{\vee}\right)$ contains at most 2 colors (see [18] $\$ 2.4$ Proposition $1)$. We say that a simple restricted root $\alpha_{i_{0}}$ is exceptional if there are two distinct simple roots $\beta_{1}$ and $\beta_{2}$ in $R_{G}$ such that: 1) $\beta_{1}-\theta\left(\beta_{1}\right)=\beta_{2}-\theta\left(\beta_{2}\right)=\alpha_{i_{0}} ; 2$ ) either $\theta\left(\beta_{1}\right) \neq-\beta_{2}$ or $\theta\left(\beta_{1}\right)=-\beta_{2}$ and $\left(\beta_{1}, \beta_{2}\right) \neq 0$. In this case we say that also $\alpha_{i_{0}}^{\vee}, \theta$ and all the equivariant embeddings of $G / H$ are exceptional. If $\alpha_{i_{0}}$ is exceptional then the simple roots $\beta_{1}$ and $\beta_{2}$ are uniquely defined by the previous properties. If $G / H$ is exceptional, then $\rho$ is not injective. Moreover, if $H=N_{G}\left(G^{\theta}\right)$, then $\rho^{-1}\left(\alpha_{i}^{\vee}\right)$ contains two colors if and only if $\alpha_{i}^{\vee}$ is exceptional. We say that $G / H$ contains a Hermitian factor if the center of $[G, G]^{\theta}$ has positive dimension. If $G / H$ does not contain a Hermitian factor then $\rho$ is injective (see [18] $\S 2.4$ Proposition 1). If $\rho$ is injective, we denote by $D_{\alpha} \vee$ the unique color contained in $\rho^{-1}\left(\alpha^{\vee}\right)$.

Remark 1. One can suppose that $G$ is the product of a connected, semisimple, simply connected group and of a central split torus in the following way. Let $\pi: \widetilde{G} \rightarrow[G, G]$ be the universal cover of the derived group of $G$. We consider the group $G^{\prime}=\widetilde{G} \times\left(T^{1} \cap Z(G)^{0}\right)$ and the map $\pi^{\prime}: G^{\prime} \rightarrow G$ defined by $\pi^{\prime}(g, t)=\pi(g) \cdot t$. 
The group $G^{\prime}$ acts transitively on $G / H$ by $g \cdot x:=\pi^{\prime}(g) \cdot x$ for each $x \in G / H$ and $g \in G^{\prime}$. Thus $G / H$ is isomorphic to $G^{\prime} /\left(\pi^{\prime}\right)^{-1}(H)$. Moreover there is a unique involution $\theta^{\prime}$ of $G^{\prime}$ such that $\pi^{\prime} \theta^{\prime}=\theta \pi^{\prime}$ (see [15]). The group $\left(\pi^{\prime}\right)^{-1}(H)$ contains and normalizes $\left(\pi^{\prime}\right)^{-1}\left(G^{\theta}\right)$, hence it normalizes also $\pi^{-1}\left([G, G]^{\theta}\right)^{0}=(\widetilde{G})^{\theta^{\prime}}$. Thus $\left(\pi^{\prime}\right)^{-1}(H)$ contains and normalizes $\left(G^{\prime}\right)^{\theta^{\prime}}$, so $G^{\prime} /\left(\pi^{\prime}\right)^{-1}(H)$ is a $G^{\prime}$-symmetric variety. We can also suppose that the stabilizer of the base point $x_{0}$ in $Z(G)^{0}$ is the subgroup of element of order 2 . Indeed, it is sufficient to consider $\widetilde{G} \times T^{\prime \prime}$, where $T^{\prime \prime}$ is a torus such that $T^{\prime \prime} /\left\{t \in T^{\prime \prime} \mid t^{2}=i d\right\}$ is isomorphic to $\left(T^{1} \cap Z(G)^{0}\right) /\left(T^{1} \cap\right.$ $\left.Z(G)^{0} \cap H\right)$.

In following, unless explicitly stated, we assume that $G$ is as in Remark 1.

We say that $\theta$ is decomposable if $G / G^{\theta}$ is the product of (non trivial) symmetric homogeneous varieties, up to quotient by a finite group. Otherwise we say that $\theta$ is indecomposable. If $\theta$ is indecomposable and $G / H$ contains a Hermitian factor, we will say that $G / H$ is Hermitian. If $\theta$ is indecomposable, we have the following possibilities: 1) $G$ is an one-dimensional torus; 2) $G$ is simple and 3) $G=\dot{G} \times \dot{G}$ with $\dot{G}$ simple and $\theta$ defined by $\theta(x, y)=(y, x)$. In the last case $G / G^{\theta}$ is isomorphic to $\dot{G}$ and is not Hermitian. If $G$ is semisimple, then $\theta$ is indecomposable if and only if $R_{G, \theta}$ is irreducible. For a classification of the indecomposable involutions see [17] $\S 26$. If $G / H$ is Hermitian, then $Z\left(G^{\theta}\right)$ has dimension 1 and $R_{G, \theta}$ has type $B C_{l}, C_{l}$, $B_{2}$ or $A_{1}$. Suppose $G$ semisimple (simply connected) and $\theta$ indecomposable, then $\rho^{-1}\left(\alpha_{i}^{\vee}\right)$ contains two colors if and only if $G / H$ is Hermitian, $H=G^{\theta}$ and $\alpha_{i}^{\vee}$ is short (see [17] pages 177-178).

Let $X$ be a simple symmetric variety with closed orbit $Y$. One can show that there is a unique affine $B$-stable open set $X_{B}$ that intersects $Y$ and is minimal for this property. Moreover the complement $X \backslash X_{B}$ is the union of the $B$-stable prime divisors not containing $Y$ (see [3] $\S 2.2$ Proposition). The stabilizer $P$ of $X_{B}$ is a parabolic subgroup containing $B$. There is a Levi subgroup $L^{\prime}$ of $P$ such that the $P$-variety $X_{B}$ is the product $R_{u} P \times Z$ of the unipotent radical of $P$ and of an affine $L^{\prime}$-spherical variety $Z$ (see [3] $§ 2.3$ Théorème). We can choose $L^{\prime}$ and $Z$ so that $x_{0}$ belongs to $Z$. Let $L$ be the standard Levi subgroup of $P$. If $X$ is not exceptional, we will see that $Z$ is a symmetric variety and that $L^{\prime}$ can be chosen equal to $L$. Notice that $P$ is the stabilizer of $\bigcup_{D \in D(G / H) \backslash D(X)} D$. Given an union $D_{i_{1}} \cup \ldots \cup D_{i_{s}}$ of colors, we denote its stabilizer by $P\left(\left\{D_{i_{1}}, \ldots, D_{i_{s}}\right\}\right)$ or by $P_{G}\left(\left\{D_{i_{1}}, \ldots, D_{i_{s}}\right\}\right)$. Given a root $\beta$, let $U_{\beta}$ be the unipotent one-dimensional subgroup of $G$ corresponding to $\beta$. Given $\mu \in \chi_{*}(T) \otimes \mathbb{Q} \equiv \chi(T) \otimes \mathbb{Q}$, we denote by $P(\mu)$ the parabolic subgroup of $G$ generated by $T$ and by the subgroups $U_{\alpha}$ corresponding to the roots $\alpha$ such that $(\alpha, \lambda) \geq 0$. Given a subgroup $K$ of $G$, we use the notation $H_{K}$ (respectively $B_{K}$ ) for the intersection $K \cap H$ (respectively $K \cap B$ ).

Now, we want to describe the groups $P=P(I)$, where $I \subset D(G / H)$. Observe that there is a Levi subgroup $L^{\prime}$ of $P$ such that $P / H_{P}=R_{u} P \times L^{\prime} / H_{L^{\prime}}$. Indeed, there is a Levi subgroup $L^{\prime \prime}$ of $P \times \mathbb{C}^{*}$ such that $P \times \mathbb{C}^{*} / H_{P} \times\{ \pm 1\}=R_{u} P \times$ $\left(L^{\prime \prime} \cap P\right) / H_{L^{\prime \prime} \cap P} \times \mathbb{C}^{*} /\{ \pm 1\}$, because of [3] $\S 2.3$ Théorème applied to an equivariant embedding $Z$ of $G \times \mathbb{C}^{*} / H \times\{ \pm 1\}$ such that $D(Z) \equiv D(G / H) \backslash I$ (we have a one-toone correspondence between $D(G / H)$ and $D\left(G \times \mathbb{C}^{*} / H \times\{ \pm 1\}\right)$, which associates to each $D \in D(G / H)$ its $\mathbb{C}^{*}$-span in $\left.G \times \mathbb{C}^{*} / H \times\{ \pm 1\}\right)$.

To describe $P(I)$, it sufficient to consider the case where $G$ is semisimple, $H=$ $G^{\theta}, \theta$ is indecomposable and $I=\{D\}$. Indeed $P\left(D_{i_{1}}, \ldots, D_{i_{s}}\right)$ is equal to the intersections of the $P\left(D_{i}\right)$. Moreover if $(G, \theta)=\left(G_{1}, \theta\right) \times\left(G_{1}, \theta\right)$ and $D$ is a color of $G / H$, then there is either a $B_{G_{1}}$-stable divisor $D_{1}$ of $G_{1} / G_{1}^{\theta}$ or a $B_{G_{2}}$-stable divisor $D_{2}$ of $G_{2} / G_{2}^{\theta}$ such that $D$ is the image of $D_{1} \times G_{2} / G_{2}^{\theta}$, respectively of $G_{1} / G_{1}^{\theta} \times D_{2}$, in $G / H$; moreover $P(D)$ is $P_{G_{1}}\left(D_{1}\right) \times G_{2}$, respectively $G_{1} \times P_{G_{2}}\left(D_{2}\right)$. Notice that $D_{1}$, respectively $D_{2}$, may be not prime. 
We begin with some recalls from [18] (see also [17] pages 177-178). Suppose by simplicity that $G$ is semisimple, write $D\left(G / G^{\theta}\right)=\left\{D_{1}, \ldots, D_{m}\right\}$ and let $\pi: G \rightarrow$ $G / G^{\theta}$ be the canonical projection. For any $i$, the ideal of $\pi^{-1}\left(D_{i}\right)$ is principal because we have supposed $G$ simply connected; let $f_{i}$ be a generator of $\pi^{-1}\left(D_{i}\right)$. Let $\mathcal{P}_{+} \subset \mathbb{C}[G]^{*}$ the multiplicative monoid constituted by the commune eigenvectors to $P$ (with respect to the left translation) and to $G^{\theta}$ (with respect to the right translation) and let $\mathcal{P}_{+}^{G^{\theta}}$ be the subset of $\mathcal{P}_{+}$constituted by the $G^{\theta}$-invariant vectors. Up to normalization, the vectors $\left\{f_{i}\right\}$ forms a basis of $\mathcal{P}_{+}$. We can index the colors so that $f_{i}$ belongs to $\mathcal{P}_{+}^{G^{\theta}}$ if and only if $i \leq r$; in particular the $G^{\theta}$-eigenvalue $\chi_{i}$ of $f_{j}$ is trivial if and only if $i \leq r$. Here $r=m-2 \operatorname{rank} \chi\left(G^{\theta}\right)$. We can also suppose that $\chi_{r+j}=-\chi_{r+j+\operatorname{rank}} \chi\left(G^{\theta}\right)$ for each $j=1, \ldots$, rank $\chi\left(G^{\theta}\right)$. The monoid $\mathcal{P}_{+}^{G^{\theta}}$ is free with basis $\left\{f_{1}, \ldots, f_{r}, f_{r+1} f_{r+\operatorname{rank} \chi}\left(G^{\theta}\right)+1, \ldots, f_{r+\operatorname{rank} \chi\left(G^{\theta}\right)} f_{m}\right\}$. The map, which associates to any $f \in \mathcal{P}_{+}$its $P$-weight $\omega(f)$, gives an isomorphism between $\mathcal{P}_{+}^{G^{\theta}}$ and the set of spherical weights. (We remark that in [18] is used a lightly different map). Observe that $P\left(D_{i}\right)$ is equal to the stabilizer of the line $\mathbb{C} f_{i}$.

Suppose now $\theta$ indecomposable, $G$ semisimple and $I=\{D\}$. If $G / G^{\theta}$ is not Hermitian, then $\mathcal{P}_{+}^{G^{\theta}}=\mathcal{P}_{+}$and $\omega\left(f_{1}\right), \ldots, \omega\left(f_{m}\right)$ are the fundamental weights of $R_{G, \theta}$; thus the stabilizer of $D_{\alpha_{i}^{\vee}}$ is $P\left(\omega_{i}\right)$. In this case, the colors of $G / H$ are in oneto-one correspondence with the colors of $G / G^{\theta}$. More generally, if $D \in D\left(G / G^{\theta}\right)$ is the unique color in $\rho^{-1}(\rho(D))$, then $\omega\left(f_{i}\right)$ belongs to $\mathcal{P}_{+}^{G^{\theta}}$ and $P(\{D\})$ is $P\left(\omega\left(f_{i}\right)\right)$; moreover $\pi^{-1}(\pi(D))$ is $D$, so also $P(\{\pi(D)\})$ is $P\left(\omega\left(f_{i}\right)\right)$ (here $\pi$ is the canonical projection $\left.G / G^{\theta} \rightarrow G / H\right)$.

Next, we consider the colors $D$ of $G / G^{\theta}$ such that $\rho^{-1}(\rho(D)) \neq\{D\}$. Supposing $\theta$ indecomposable, there is an exceptional root $\alpha_{i_{0}}$ if and only if $G / G^{\theta}$ is Hermitian and $R_{G, \theta}$ has type $B C_{l}$. In this case $H=G^{\theta}=N_{G}\left(G^{\theta}\right)$ and $2 \alpha_{i_{0}}$ is a restricted root; in particular $\alpha_{i_{0}}^{\vee}$ is the short simple restricted coroot and $\rho^{-1}\left(\alpha_{i_{0}}^{\vee}\right)$ contains two colors, namely $D_{r+1}$ and $D_{r+2}$. Write $\alpha_{i_{0}}=\beta_{i_{1}}-\theta\left(\beta_{i_{1}}\right)=\beta_{i_{2}}-\theta\left(\beta_{i_{2}}\right)$ as in the definition of exceptional root. The weight of $f_{r+1} f_{r+2}$ is $\omega_{i_{0}}=\varpi_{i_{1}}+\varpi_{i_{2}}$. Thus $f_{r+1}$ and $f_{r+2}$ have weights respectively $\varpi_{i_{1}}$ and $\varpi_{i_{2}}$; the stabilizers of the corresponding colors are respectively $P\left(\varpi_{i_{1}}^{\vee}\right)$ and $P\left(\varpi_{i_{2}}^{\vee}\right)$. There is an automorphism of $G$, fixing $T$ and $B$, which exchange $P\left(\varpi_{i_{1}}^{\vee}\right)$ with $P\left(\varpi_{i_{2}}^{\vee}\right)$; moreover, one can show, by the Iwasawa decomposition (see [17] p.168), that this automorphism fixes $G^{\theta}$, so it induces an automorphism of $G / G^{\theta}$ exchanging $D_{1}$ with $D_{2}$. For this case, see also [6] $\S 4$.

If $G / H$ is Hermitian and non exceptional, then the restricted root lattice has index two in the lattice generated by the spherical weights; the inverse image of $\alpha_{i}^{\vee}$ contains two colors if and only if $H=G^{\theta}$ and $\alpha_{i}^{\vee}$ is short (see [17] page 177178). Therefore the weight of $f_{r+1} f_{r+2}$ is $\omega_{1}=2 \varpi_{1}$ if $l$ is at most 2 and $\omega_{l}=2 \varpi_{l}$ otherwise. The vectors $f_{r+1}$ and $f_{r+2}$ have the same weight, namely $\varpi_{1}$ if $l \leq 2$ and $\varpi_{2}$ otherwise. Thus the colors corresponding to $f_{r+1}$ and $f_{r+2}$ have the same stabilizer: respectively $P\left(\omega_{1}\right)$ if $l \leq 2$ and $P\left(\omega_{l}\right)$ otherwise. Moreover these colors have the same image in $G / N_{G}\left(G^{\theta}\right)$. Hence the stabilizer of a color $D$ of $G / G^{\theta}$ is equal to the stabilizer of the image of $D$ in $G / N_{G}\left(G^{\theta}\right)$.

Now we list the exceptional indecomposable involutions. We have three possibilities: 1) $(G, \theta)$ has type $A I I I$ and $G / G^{\theta}$ is isomorphic to $S L_{n+1} / S\left(L_{l} \times\right.$ $L_{n+1-l}$ ) (with $\left.n \neq 2 l-1\right) ; 2$ ) $(G, \theta)$ has type $D I I I$ and $G / G^{\theta}$ is isomorphic to $\left.S O_{4 l+2} / G L_{2 l+1} ; 3\right)(G, \theta)$ has type $E I I I$ and $G / G^{\theta}$ is isomorphic to $E_{6} / D_{5} \times \mathbb{C}^{*}$. The possibilities for the stabilizer of a color $D$ with $\rho(D)$ exceptional are, respectively: 1) $P\left(\widetilde{\omega}_{l}\right)$ and $P\left(\widetilde{\omega}_{n+1-l}\right)$; 2) $P\left(\widetilde{\omega}_{2 l}\right)$ and $P\left(\widetilde{\omega}_{2 l+1}\right)$; 3) $P\left(\widetilde{\omega}_{1}\right)$ and $P\left(\widetilde{\omega}_{6}\right)$. 


\section{Smoothness of SymmetriC VARIETIES}

In this section we want to classify the smooth symmetric varieties. Clearly we can reduce ourselves to studying the simple ones. Let $X$ be a simple symmetric variety with closed orbit $Y$. Recall that $X_{B} \equiv R_{u} P \times Z$, where $P=P(D(G / H) \backslash D(X))$ and $Z$ is a $L^{\prime}$-spherical affine variety for an appropriate Levi subgroup $L^{\prime}$ of $P$; we have denoted by $L$ the standard Levi subgroup of $P$.

Lemma 2.1. The homogeneous variety $L^{\prime} / H_{L^{\prime}}$ is affine; in particular $H_{L^{\prime}}$ is reductive.

Proof. $R_{u} P \times\left(L^{\prime} / H_{L^{\prime}}\right)$ is the intersection of the two affine open sets $X_{B}$ and $G / H$, thus it is affine. Hence $L^{\prime} / H_{L^{\prime}}$ is affine and $H_{L^{\prime}}$ is reductive.

Lemma 2.2. If $\rho^{-1}\left(\alpha^{\vee}\right)$ is contained in $D(X)$ for each exceptional coroot $\alpha^{\vee}$ in $\rho(D(X))$, then the group $L$ is $\theta$-stable and $L / H_{L}$ is a symmetric space; moreover we can choose $L^{\prime}$ equal to $L$. In particular, $L$ is $\theta$-stable if $X$ is not exceptional.

Proof of Lemma 2.2. Observe that $P$ contains the stabilizer $\widetilde{P}$ of $B H / H$. Indeed the colors are exactly the prime divisors of $G / H$ stabilized by $\widetilde{P}$. Let $\widetilde{\lambda} \in \chi_{*}(T)$ such that $\widetilde{P}=P(\widetilde{\lambda}) ; \tilde{\lambda}$ is a subgroup of $T^{1}$ (see [18] 2.2 ), so $(\tilde{\lambda}, \beta)=0$ for every root $\beta$ in $R_{G}^{0}$. Hence $U_{\beta} \subset \widetilde{P} \subset P$ and $R_{L}$ contains $R_{G}^{0}$. Moreover $R_{L}$ is stabilized by the involution $\iota$, because $\beta_{1}-\theta\left(\beta_{1}\right)=\beta_{2}-\theta\left(\beta_{2}\right)$ if and only if $\beta_{1}=\beta_{2}$ or $\beta_{1}=\iota\left(\beta_{2}\right)$. Thus $R_{L}$ is stabilized by $\theta=-\varpi_{0} \iota$, where $\varpi_{0}$ is the longest element of the Weyl group of $R_{G}^{0}$. Therefore $P \cap \theta(P)=L, H_{L}=H_{P}$ and we can suppose $L=L^{\prime}$. Notice that $L^{\theta} \subset L \cap H \subset N_{L}\left(G^{\theta}\right) \subset N_{L}\left(L^{\theta}\right)$.

Under the hypotheses of the previous lemma, $R_{L}$ is generated by $R_{G}^{0}$ and by the simple root $\beta$ in $R_{G}^{1}$ such that $\rho^{-1}\left((\beta-\theta(\beta))^{\vee}\right)$ is contained in $D(X)$ (see the description of $P$ in the previous section). In particular, $R_{L, \theta}^{\vee}=\rho(D(X))$ if $G / H$ has not Hermitian factors.

We are mostly interested in the smooth complete symmetric varieties, so we can reduce ourselves to studying the simple affine varieties with a $L^{\prime}$-fixed point.

Lemma 2.3. If $Y$ is projective, then there is a unique point in $Z$ fixed by $L^{\prime}$.

Proof of Lemma 2.3. Notice that $Z^{\prime} \cap Y$ is one point $x$ and is $L^{\prime}$-stable. Moreover $x$ is unique because every affine spherical variety is simple.

The hypothesis of the previous lemma is equivalent to the condition $\operatorname{dim} C(X)$ $=\operatorname{rank} G / H$. Now, we prove that $X$ is not smooth if $L$ is not $\theta$-stable.

Theorem 2.1. Let $X$ be a simple smooth symmetric variety with open orbit $G / H$ and suppose that the closed orbit $Y$ is projective. Then $\rho^{-1}\left(\alpha^{\vee}\right)$ is contained in $D(X)$ for each exceptional coroot $\alpha^{\vee}$ in $\rho(D(X))$. In particular, $L$ is $\theta$-stable.

Observe that $X$ is smooth if and only if $Z$ is smooth. Moreover $Z$ is smooth if and only if it is a $L^{\prime}$-representation. Indeed the affine $L^{\prime}$-variety $Z$ can be embedded in a $L^{\prime}$-representation and one can shift the fixed point in 0 . Moreover, up to taking a subrepresentation, we can identify $Z$ with this representation because of Luna's fundamental lemma (see [10] II.2). To prove the lemma, it is sufficient to show that there is not a representation of dimension $\operatorname{dim} L^{\prime} / H_{L^{\prime}}$ containing a vector $v$ with stabilizer $H_{L^{\prime}}$. Observe that we can work with a quotient of $L^{\prime}$ by a finite central subgroup $K$ contained in $H_{L^{\prime}}$. Indeed, if $Z$ is a $L^{\prime}$-representation with the previous properties, $K$ acts trivially on $Z$, so $Z$ is a $\left(L^{\prime} / K\right)$-representation of dimension $\left(L^{\prime} / K\right) /\left(H_{L^{\prime}} / K\right)$ and $\operatorname{Stab}_{\left(L^{\prime} / K\right)}(v)=\left(H_{L^{\prime}} / K\right)$. Remark that $L^{\prime} / K$ is not plus a subgroup of $G$, however we can restrict to study $Z$, "forgetting" the embedding of $Z$ in $X$. We want reduce to study varieties $L^{\prime} / H_{L^{\prime}}$ with the following property: 
[*] There are an indecomposable exceptional involution $\left(G^{\prime}, \theta^{\prime}\right)$ and a color $D$ such that $\rho(D)$ is exceptional, $L^{\prime}$ is a Levi subgroup of the stabilizer $P^{\prime}=P_{G^{\prime}}(D), H_{L^{\prime}}=\left(L^{\prime}\right)^{\theta^{\prime}}$ and $P^{\prime} /\left(P^{\prime}\right)^{\theta^{\prime}} \equiv R_{u} P^{\prime} \times$ $L^{\prime} / H_{L^{\prime}}$.

Lemma 2.4. We can choose $L^{\prime}$ isomorphic, up to quotient by a finite central group in $H_{L^{\prime}}$, to a product $\prod_{i=0}^{s} L_{i}$ so that $L^{\prime} / H_{L^{\prime}} \equiv \prod L_{i} / H_{L_{i}}$, where $L_{i} / H_{L_{i}}$ is a symmetric variety if $s=0$ and satisfies [*] otherwise.

Proof of Lemma 2.4. First, we reduce to the case of an indecomposable involution. Write $(G, \theta)$ as $\prod\left(G_{i}, \theta\right)$, where $G_{0} \cap L$ is $\theta$-stable and the $\left(G_{i}, \theta\right)$ with $i>0$ are indecomposable exceptional involutions such that $G_{i} \cap L$ is not $\theta$-stable. Then $G / H=G_{0} / H_{G_{0}} \times \prod_{i>0} G_{i} / G_{i}^{\theta}$. Indeed $G_{i}^{\theta}=N_{G_{i}}\left(G_{i}^{\theta}\right)$ for each $i>0$, thus $N_{G}\left(G^{\theta}\right)$ is generated by $G^{\theta}$ and $H_{G_{0}}$. Thus it sufficient to prove the lemma for the $G_{i} / G_{i}^{\theta}$ with $i>0$. Finally, suppose $\theta$ an indecomposable exceptional involution and write $P=P(I)$ with $I \subset D(G / H)$. Let $D$ be a color in $I$ such that $\rho(D)$ is exceptional. If $I$ contains at least two colors, let $\widetilde{L}$ be the standard Levi subgroup of $P(I \backslash\{D\})$, let $\bar{L}$ be the quotient of $\widetilde{L}$ by $Z([\widetilde{L}, \widetilde{L}])$ and let $\bar{P}$ be the quotient of $P(I) \cap \widetilde{L}$ by $Z([\widetilde{L}, \widetilde{L}])$. We have $P(I)=R_{u} P(I \backslash\{D\}) \times(P(I) \cap \widetilde{L})$ and $P(I) / P(I)^{\theta}=R_{u} P(I \backslash\{D\}) \times(P(I) \cap \widetilde{L}) /(P(I) \cap \widetilde{L})^{\theta}=R_{u} P(I \backslash\{D\}) \times \bar{P} / \bar{P}^{\theta} ;$ moreover $\widetilde{L} / \widetilde{L}^{\theta}=\bar{L} / \bar{L}^{\theta}=[\bar{L}, \bar{L}] /[\bar{L}, \bar{L}]^{\theta} \times Z(\bar{L}) / Z(\bar{L})^{\theta}$. On the other hand, there is a Levi subgroup $\bar{L}^{\prime}$ of $\bar{P} \cap[\bar{L}, \bar{L}]$ such that $\bar{P} / \bar{P}^{\theta}=R_{u} \bar{P} \times \bar{L}^{\prime} /\left(\bar{L}^{\prime}\right)^{\theta} \times Z\left(\bar{L}^{\prime}\right) / Z\left(\bar{L}^{\prime}\right)^{\theta}$. Thus we can choose $L^{\prime}$ as the product of $Z(\widetilde{L})^{0}$ with the inverse image of $\bar{L}^{\prime}$ in $\widetilde{L}$. Notice that $([\bar{L}, \bar{L}], \theta)$ is indecomposable and exceptional; moreover $\bar{P} \cap[\bar{L}, \bar{L}]$ is the stabilizer of $D \cap\left([\bar{L}, \bar{L}] /[\bar{L}, \bar{L}]^{\theta}\right)$ in $[\bar{L}, \bar{L}]$.

We know that given a semisimple group $G$, a symmetric variety $G / H$ and an irreducible $G$-representation $V$, then $V^{H}$ has dimension at most 1; moreover, if $\operatorname{dim} V^{H}=1, V$ has dimension at least $\operatorname{dim} G / H+1$. We want to prove a similar result for the varieties which satisfy $[*]$. Such varieties have dimension $\operatorname{dim} G /(G)^{\theta}-\operatorname{dim} R_{u} P$, because $P / H_{P}=R_{u} P \times L^{\prime} / H_{L^{\prime}}$. Explicitly $\operatorname{dim} L^{\prime} /\left(L^{\prime}\right)^{\theta}$ is equal, respectively, to $n l-l^{2}+l$ if $G / G^{\theta}=S L_{n+1} / S\left(L_{l} \times L_{n+1-l}\right)$, to $2 l^{2}+l$ if $G / G^{\theta}=S O_{4 l+2} / G L_{2 l+1}$ and to 16 if $G / G^{\theta}=E_{6} / D_{5} \times \mathbb{C}^{*}$. In particular $L^{\prime} /\left(L^{\prime}\right)^{\theta}$ has dimension at least 2 . We consider separately the case where $\operatorname{dim} L^{\prime} /\left(L^{\prime}\right)^{\theta}=2$, because in such case $L^{\prime} /\left(L^{\prime}\right)^{\theta}$ is isomorphic to a symmetric variety.

Lemma 2.5. If $L^{\prime} / H_{L^{\prime}}$ has dimension 2, then it is isomorphic to $\mathrm{SL}_{2} / \mathrm{SO}_{2}$. Thus, a non-trivial $L^{\prime}$-representation $V$ with $V^{H_{L^{\prime}}} \neq 0$ has dimension at least 3. Moreover, there are neither a smooth affine embedding of $\mathrm{SL}_{2} / \mathrm{SO}_{2}$ nor a smooth affine embedding of $S L_{2} \times \mathbb{C}^{*} / S O_{2} \times\{ \pm 1\}$.

Proof of Lemma 2.5. If $\operatorname{dim} L^{\prime} / H_{L^{\prime}}=2$, then $G / H$ is isomorphic to $S L_{3} /$ $S\left(L_{1} \times L_{2}\right)$ and $P$ is either $P\left(\varpi_{1}^{\vee}\right)$ or $P\left(\varpi_{2}^{\vee}\right)$; thus $L^{\prime}$ is isomorphic to $G L_{2}$. By the Lemma 2.2, $H_{L^{\prime}}^{0}$ must be a maximal torus, hence $L^{\prime} / H_{L^{\prime}}$ is a symmetric variety isomorphic either to $S L_{2} / S O_{2}$ or to $S L_{2} / N_{S L_{2}}\left(S O_{2}\right)$. Observe that a spherical $S L_{2}$-representation has dimension at least 3 . In this case the representation is $S^{2} \mathbb{C}^{2}$ and is an embedding of $S L_{2} \times \mathbb{C}^{*} / N_{S L_{2}}\left(S O_{2}\right) \times\{ \pm 1\}$, where $\mathbb{C}^{*}$ acts freely over $\mathbb{C}^{2}$. We have only to prove that $L^{\prime} / H_{L^{\prime}}$ is isomorphic to $S L_{2} / S O_{2}$. Suppose by contradiction that $H_{L^{\prime}}$ is isomorphic to the almost direct product $N_{S L_{2}}\left(S O_{2}\right) \cdot \mathbb{C}^{*} i d$ and let $\sigma$ be an involution of $G L_{2}$ such that $G L_{2}^{\sigma}$ is $H_{L^{\prime}}^{0}$. Let $T^{\prime}$ be a maximally split torus of $G L_{2}$ with respect to $\sigma$ and let $S^{\prime}=T^{\prime} / H_{T^{\prime}}$, then $\chi\left(T^{\prime}\right) / \chi\left(S^{\prime}\right)$ contains an element of order 2 . Indeed $\chi\left(S^{\prime}\right)$ is generated by $2 \beta$ and $\chi\left(T^{\prime}\right)$ contains $\beta$ (here $\beta$ is a root of $\left.G L_{2}\right)$. We can identify $\mathbb{C}\left(L^{\prime} / H_{L^{\prime}}\right)^{\left(B_{L^{\prime}}\right)} / \mathbb{C}^{*}$ with $\mathbb{C}(G / H)^{(B)} / \mathbb{C}^{*}$, thus we can identify $\chi(S)$ with $\chi\left(S^{\prime}\right)$ as subset of $\chi(B)$; moreover we can identity both 
$\chi(T)$ and $\chi\left(T^{\prime}\right)$ with $\chi(B)$. Thus also $\chi(T) / \chi(S)$ contains an element of order 2 . But $\chi(S)$ is equal to $\varpi_{1}+\varpi_{2}$, so $\chi(T) / \chi(S)=\mathbb{Z}\left(\varpi_{1}+\chi(S)\right) \cong \mathbb{Z}$, a contradiction. (Here $\varpi_{1}$ and $\varpi_{2}$ are the fundamental weights of $\left.S L_{3}\right)$.

Lemma 2.6. Assume that $L^{\prime} /\left(L^{\prime}\right)^{\theta}$ has dimension greater than 2, then there is a subgroup $K$ of $L^{\prime}$ isomorphic to $L \cap \theta(L)$ in such way that $L^{\theta} \subset L \cap \theta(L)$ is isomorphic to a subgroup $K^{\prime}$ of $K^{\theta}$. Let $V$ be a $L^{\prime}$-representation which contains a vector $v$ fixed by $K^{\theta}$. Then the stabilizer of $v$ in $[K, K]$ is equal either to $[K, K]$ or to $[K, K]^{\prime}:=[K, K] \cap K^{\prime}$.

Proof of Lemma 2.6. Write $L^{\prime}=g L g^{-1}$; we have $L^{\theta} \subset P^{\theta}=\left(L^{\prime}\right)^{\theta}$, because $H=G^{\theta}$. Knowing the classification of indecomposable exceptional involution, one can verify that there is an automorphism $\varphi$ of $L^{\prime}$ that send $g L^{\theta} g^{-1}$ onto $L^{\theta}$. Let $K$ be the image of $g(L \cap \theta(L)) g^{-1}$ by $\varphi$, then $K^{\theta}$ contains $K^{\prime}:=L^{\theta}=\varphi\left(g L^{\theta} g^{-1}\right)$. The last affirmation follows because $[K, K] /[K, K]^{\prime}$ is a symmetric variety.

Remark that $K / K^{\prime}$ is a symmetric variety.

Lemma 2.7. Let $(G, \theta)$ be an indecomposable exceptional involution, let $D$ be a color such that $\rho(D)$ is exceptional, let $P$ be the stabilizer of $D$ and let $L^{\prime}$ be a Levi subgroup of $P$ such that $P / H_{P}=R_{u} P \times L^{\prime} / H_{L^{\prime}}$. Suppose that $\operatorname{dim} L^{\prime} / H_{L^{\prime}}>2$ and let $K$, respectively $K^{\prime}$, be as in the Lemma 2.6. Let $V$ be a non-trivial irreducible $L^{\prime}$-representation such that $V^{H_{L^{\prime}}} \neq 0$. Then:

(1) $\operatorname{dim} V \geq \operatorname{dim} L^{\prime} / H_{L^{\prime}}$ and $\operatorname{dim} V \neq \operatorname{dim} L^{\prime} / H_{L^{\prime}}+1$;

(2) if $\operatorname{dim} V=\operatorname{dim} L^{\prime} / H_{L^{\prime}}$, then $V^{H_{L^{\prime}}}$ has dimension 1 and $\left(\operatorname{Stab}_{L^{\prime}}(v)\right)^{0}$ is not reductive for any $v \in V^{H_{L^{\prime}}} \backslash\{0\}$;

(3) if there is $v \in V$ with stabilizer $H_{L^{\prime}}$, then $\operatorname{dim} V \geq \operatorname{dim} L^{\prime} / H_{L^{\prime}}+2$.

Proof of the Lemma 2.7. Observe that the first two points of the lemma imply the third one. Indeed, if $V$ is as in the last point, then $V$ cannot have dimension equal to $\operatorname{dim} L^{\prime} / H_{L^{\prime}}$ because $H_{L^{\prime}}$ is reductive by the Lemma 2.2. Notice that $K$ contains a maximal torus; moreover there are two parabolic groups $Q$ and $Q^{-}$of $L^{\prime}$, containing respectively $B_{L^{\prime}}$ and the opposite of $B_{L^{\prime}}$, such that $Q \cap Q^{-}=K$ is a Levi subgroup of both them.

For each $r$, let $\left\{e_{1}, \ldots, e_{r}\right\}$ be the canonical basis of $\mathbb{C}^{r}$. It is sufficient to study the $L^{\prime}$-representations with dimension at most $\operatorname{dim} L^{\prime} / H_{L^{\prime}}+1$. If $G / H=$ $G L_{n+1} / G L_{l} \times G L_{n+1-l}\left(=S L_{n+1} / S\left(L_{l} \times L_{n+1-l}\right)\right)$, then $L^{\prime}$ is isomorphic to $G L\left(\bigoplus_{i=1}^{n-l+1} \mathbb{C} e_{i}\right)$ $\times G L\left(\bigoplus_{i=n-l+2}^{n+1} \mathbb{C} e_{i}\right)$ in such way that $K$ corresponds to $G L\left(\bigoplus_{i=1}^{l} \mathbb{C} e_{i}\right) \times G L\left(\bigoplus_{i=l+1}^{n-l+1} \mathbb{C} e_{i}\right) \times$ $G L\left(\bigoplus_{i=n-l+2}^{n+1} \mathbb{C} e_{i}\right)$ and $K^{\prime}$ corresponds to $\Delta \times G L\left(\bigoplus_{i=l+1}^{n-l+1} \mathbb{C} e_{i}\right)$, where $\Delta$ is the diagonal in $G L\left(\bigoplus_{i=1}^{l} \mathbb{C} e_{i}\right) \times G L\left(\bigoplus_{i=n-l+2}^{n+1} \mathbb{C} e_{i}\right)$. Recall that $n>2$. Suppose first that $[K, K]$ acts trivially on a non-zero vector of $V^{H_{L^{\prime}}}$; in this case $S L\left(\bigoplus_{i=n-l+2}^{n+1} \mathbb{C} e_{i}\right)$ acts trivially on $V$. Using the fact that $\operatorname{dim} Z\left(K^{\prime}\right)=2$, it is not difficult to prove that $V^{[K, K]}$ is one-dimensional and that $Z\left(K^{\prime}\right)$ acts not trivially on it, a contradiction. Hence, both the simple factors of $[K, K]$ act not trivially on $V$ (otherwise there is $(g, g) \in \Delta$ such that $(g, g) \cdot v \neq v$ ). The representation $W:=\bigoplus_{i=1}^{n-l+1} \mathbb{C} e_{i} \otimes\left(\bigoplus_{i=n-l+2}^{n+1} \mathbb{C} e_{i}\right)^{*}$ has dimension equal to $\operatorname{dim} L^{\prime} / H_{L^{\prime}}$. If there is another $L^{\prime}$-representation, not isomorphic to $W$ as $\left[L^{\prime}, L^{\prime}\right]$-representation, over which both the simple factors of $[K, K]$ acts non-trivially and which has dimension at most $\operatorname{dim} W+1$, then either $n=2 l=4$ or $l=1$. In the first case such representation is isomorphic to $W_{1}:=\bigwedge^{2} \bigoplus_{i=1}^{3} \mathbb{C} e_{i} \otimes\left(\bigoplus_{i=4}^{5} \mathbb{C} e_{i}\right)^{*}$ (as $\left[L^{\prime}, L^{\prime}\right]$-representation); moreover it is isomorphic to $W$ as $[K, K]$-representation. In the second case, such representation is isomorphic to $W_{2}:=\bigwedge^{n-1} \bigoplus_{i=1}^{n} \mathbb{C} e_{i}$ (as $\left[L^{\prime}, L^{\prime}\right]$-representation) and we have $[K, K]=[K, K]^{\prime}=S L\left(\bigoplus_{i=2}^{n} \mathbb{C} e_{i}\right)$, thus $W_{2}$ is isomorphic, as $[K, K]$-representation, to the direct sum of a 1-dimensional 
trivial representation with the dual of the standard representation. The decomposition $W=\left(\bigoplus_{i=1}^{l} \mathbb{C} e_{i} \otimes\left(\bigoplus_{i=n-l+2}^{n+1} \mathbb{C} e_{i}\right)^{*}\right) \oplus\left(\bigoplus_{l+1}^{n-l+1} \mathbb{C} e_{i} \otimes\left(\bigoplus_{i=n-l+2}^{n+1} \mathbb{C} e_{i}\right)^{*}\right)$ is $[K, K]$-invariant; thus $W^{[K, K]^{\prime}}$ is 1-dimensional. One can show that $R_{u} Q$ stabilizes any $v \in W^{[K, K]^{\prime}} ;$ moreover $\operatorname{dim} \operatorname{Stab}_{L^{\prime}}(v)=\operatorname{dim} R_{u} Q \cdot[K, K]^{\prime}+1$. On the other hand, $\operatorname{dim} Z(K)=2$ and $Z(K) \cdot v$ is at plus, one-dimensional. Therefore $T^{\prime \prime}:=\operatorname{Stab}_{Z(K)}(v)$ is one-dimensional and the connected component of $\operatorname{Stab}_{L^{\prime}}(v)$ is the semidirect product $R_{u} Q \ltimes\left(T^{\prime \prime}[K, K]^{\prime}\right)^{0}$, so it is not reductive. Given any $U$ isomorphic, as $\left[L^{\prime}, L^{\prime}\right]$-representation, to $W$, to $W_{1}$ or to $W_{2}$, one can study in the same manner $U^{[K, K]^{\prime}}$ and $\operatorname{Stab}_{L^{\prime}}(v)$ for each $v \in U^{[K, K]^{\prime}} \backslash\{0\}$.

If $G=S O_{4 l+2}$, then $L^{\prime}$ is isomorphic to $G L\left(\mathbb{C}^{2 l+1}\right)$ in such way that $K$ corresponds to $G L\left(\bigoplus_{i=1}^{2 l} \mathbb{C} e_{i}\right) \times G L\left(\mathbb{C} e_{2 l+1}\right)$ and $K^{\prime}$ corresponds to $S p\left(\bigoplus_{i=1}^{2 l} \mathbb{C} e_{i}\right) \times$ $G L\left(\mathbb{C}_{2 l+1}\right)$. The non-trivial irreducible $L^{\prime}$-representations with dimension lesser or equal to $2 l^{2}+l+1$ are isomorphic, as $\left[L^{\prime}, L^{\prime}\right]$-representations, to $\mathbb{C}^{2 l+1}$, to $\bigwedge^{2} \mathbb{C}^{2 l+1}$, to $\bigwedge^{2 l-1} \mathbb{C}^{2 l+1}$ or to $\bigwedge^{2 l} \mathbb{C}^{2 l+1}$. Let $V$ be a $L^{\prime}$-representation which contains a nonzero vector $w$ fixed by $K^{\prime}$ and that is isomorphic, as $\left[L^{\prime}, L^{\prime}\right]$-representation, to $\mathbb{C}^{2 l+1}$. We have $\left(\mathbb{C}^{2 l+1}\right)^{[K, K]}=\left(\mathbb{C}^{2 l+1}\right)^{[K, K]^{\prime}}=\mathbb{C} e_{2 l+1}$; thus $V$ is $\mathbb{C}^{2 l+1} \otimes\left(\bigwedge^{2 l+1} \mathbb{C}^{2 l+1}\right)^{*}$ and $w$ is a multiple of $e_{2 l+1} \otimes v$, where $v$ is a non-zero vector of $\left(\bigwedge^{2 l+1} \mathbb{C}^{2 l+1}\right)^{*}$. The stabilizer of $e_{2 l+1} \otimes v$ in $L^{\prime}$ is $R_{u} Q^{-} \ltimes\left(S L\left(\bigoplus_{i=1}^{2 l} \mathbb{C} e_{i}\right) \times G L\left(\mathbb{C} e_{2 l+1}\right)\right)$. If $H_{L^{\prime}}$ fixes $e_{2 l+1} \otimes v$, then $H_{L^{\prime}}$ is isomorphic to a subgroup of $S L_{2 l} \times \mathbb{C}^{*}$ (because the restriction to $H_{L^{\prime}}$ of $\operatorname{Stab}_{L^{\prime}}\left(e_{2 l+1}\right) \rightarrow \operatorname{Stab}_{L^{\prime}}\left(e_{2 l+1}\right) / R_{u} Q^{-}$would be an isomorphism). However, one can show that there is not a reductive subgroup of $S L_{2 l} \times \mathbb{C}^{*}$, containing $S p_{2 l} \times \mathbb{C}^{*}$, which has dimension equal to $\operatorname{dim} H_{L^{\prime}}$, a contradiction. One can study in the same manner the $L^{\prime}$-representations isomorphic, as $\left[L^{\prime}, L^{\prime}\right]$-representations, to $\bigwedge^{2 l} \mathbb{C}^{2 l+1}$. On the other hand, $\bigwedge^{2} \mathbb{C}^{2 l+1}$ is isomorphic, as $[K, K]$-representation, to $\bigwedge^{2} \bigoplus_{i=1}^{2 l} \mathbb{C} e_{i} \oplus \bigoplus_{i=1}^{2 l} \mathbb{C} e_{i}$, hence $\left(\bigwedge^{2} \mathbb{C}^{2 l+1}\right)^{[K, K]^{\prime}}$ is 1-dimensional. the stabilizer in $L^{\prime}$ of any $v \in\left(\bigwedge^{2} \mathbb{C}^{2 l+1}\right)^{[K, K]^{\prime}} \backslash\{0\}$ contains $R_{u} Q$; moreover $\operatorname{dim} \operatorname{Stab}_{L^{\prime}}=$ $\operatorname{dim} R_{u} P \cdot[K, K]^{\prime}+1$. On the other hand, $T^{\prime \prime}:=\operatorname{Stab}_{Z(K)}(v)$ is one-dimensional because $Z(K) \cdot(v)$ is, at plus, one-dimensional. Therefore, the connected component of $\operatorname{Stab}_{L^{\prime}}(v)$ is the semidirect product $\left.R_{u} P \ltimes\left(T^{\prime \prime}[K, K]^{\prime}\right)\right)^{0}$, so it is not reductive. One can study $\bigwedge^{2 l-1} \mathbb{C}^{2 l+1}$ in the same manner of $\bigwedge^{2} \mathbb{C}^{2 l+1}$ (notice that $\bigwedge^{2 l-1} \mathbb{C}^{2 l+1}$ is the dual representation of $\left.\bigwedge^{2} \mathbb{C}^{2 l+1}\right)$.

If $L$ has type $E_{6}$, then, up to isogeny, $L^{\prime}$ is isomorphic to $\operatorname{Spin}_{10} \times \mathbb{C}^{*}$, in such way that $K$ corresponds to $\operatorname{Spin}_{8} \times S_{2} \times \mathbb{C}^{*}$ and $K^{\prime}$ corresponds to $\operatorname{Spin}_{7} \times \mathbb{C}^{*}$. By a dimensional count, one can show that $H_{L^{\prime}}$ is isomorphic to $K$ (one have to use the fact that $K^{\prime} \subset H_{L^{\prime}} \subset L^{\prime}$ ). Observe that the hypothesis $V^{H_{L^{\prime}}} \neq 0$ implies that $Z\left(L^{\prime}\right)$ acts trivially on $V$. The irreducible non-trivial $\operatorname{Spin}_{10}$-representations with dimension at most 17 are the half-spin representations and the first fundamental representation $\mathbb{C}^{10}$. One can easily show that none of such representation contains a vector fixed by $H_{L^{\prime}}$.

Proof of Theorem 2.1. We work with a quotient $\widetilde{L}$ of $L^{\prime}$ by a central subgroup in $H_{L^{\prime}}$, so that we can write $\widetilde{L}=\prod L_{j}$ as in the Lemma 2.4. We denote by $H_{\widetilde{L}}$ the image of $H_{L^{\prime}}$ in $\widetilde{L}$. We want to reduce ourselves to the case where $\widetilde{L} / \widetilde{L}^{\theta}$ is a product of a variety satisfying $[*]$ and, eventually, a 1-dimensional torus. Suppose $Z$ smooth and write $Z=\bigoplus_{h=1}^{m} V\left(\lambda_{h}\right)$. Observe that $\widetilde{L}$ has a dense orbit in each $V\left(\lambda_{h}\right)$, say $\widetilde{L} \cdot v_{h}$; we can also suppose $\sum_{h=1}^{m} v_{i}=x_{0}$. Moreover $Z(\widetilde{L})^{0} \cdot v_{h}$ is contained in $\mathbb{C}^{*} v_{h}$, so $\operatorname{dim} \widetilde{L} \cdot v_{h} \leq \operatorname{dim}\left([\widetilde{L}, \widetilde{L}] \cdot v_{h}\right)+1$. Up to re-index, we can suppose that $L_{1}$ does not act trivially on $V\left(\lambda_{1}\right)$. Write $\lambda_{i}=\sum \mu_{i, j}$, where $\mu_{i, j}$ is a $L_{j}$-weight for each $i$ and $j$, so $V\left(\lambda_{i}\right)$ is isomorphic to $\bigotimes_{j} V\left(\mu_{i, j}\right)$. Thus $V\left(\mu_{i, j}\right)$ contains a $H_{\widetilde{L}}$-fixed vector $v$ for each $i$ and $j$; hence, by the Lemmas 2.5 and $2.7, \operatorname{dim} V\left(\mu_{i, j}\right) \neq 2$ for each $j$. Let $I$ be the set $\left\{j \mid \operatorname{dim} V\left(\mu_{1, j}\right)>1\right\}$. If $I$ contains at least 2 elements, then 
$\operatorname{dim} V\left(\lambda_{1}\right)=\prod_{j \in I} \operatorname{dim} V\left(\mu_{i, j}\right)>\sum_{j \in I} \operatorname{dim} V\left(\mu_{i, j}\right)+1 \geq \sum_{j \in I} \operatorname{dim}\left(L_{j} / H_{L_{j}}\right)+$ $1 \geq \operatorname{dim} \widetilde{L} v_{1}$, a contradiction. Thus $V\left(\sum \mu_{1, j}\right)=V\left(\mu_{1,1}\right) \otimes V\left(\sum_{j \neq 1} \mu_{1, j}\right)$, where $\operatorname{dim} V\left(\sum_{j \neq 1} \mu_{1, j}\right)=1$. We have two possibilities: either $V\left(\lambda_{1}\right)$ is a smooth affine embedding of $L_{1} \cdot v_{1}$ or there is a one-dimensional torus $T_{1} \subset \prod_{i \neq 1} L_{i}$, such that $V\left(\lambda_{1}\right)$ is a smooth affine embedding of $\left(L_{1} T_{1}\right) \cdot v_{1}$; in particular the dimension of $V\left(\lambda_{1}\right)$ is at most $\operatorname{dim} L_{1} / H_{L_{1}}+1$. This fact implies, by the Lemma 2.7 , that $\operatorname{dim} L_{1} / H_{L_{1}}$ is 2 .

Finally, suppose that $\operatorname{dim} L_{i} / H_{L_{i}}=2$ for each $i>0$. By the Lemma 2.5, $\operatorname{dim} V\left(\lambda_{i}\right)=\operatorname{dim}\left[L^{\prime}, L^{\prime}\right] v_{i}+1$ for each $i$. Moreover we can suppose that, for each $i>0, L_{i}$ acts non-trivially on $V\left(\lambda_{j}\right)$ if and only if $i=j$; otherwise $L^{\prime} \cdot \sum_{1}^{m} v_{i}$ is not dense. We can identify $L_{1} / H_{L_{1}}$ with $G L_{2} /\left(\mathbb{C}^{*}\right)^{2}$ and $V\left(\mu_{1,1}\right)$ with $S^{2} \mathbb{C}^{2}$. Let $n \in N_{S L_{2}}\left(\left(\mathbb{C}^{*}\right)^{2}\right) \backslash\left(\mathbb{C}^{*}\right)^{2}$, then $n \cdot v_{1}=-v_{1}$. The center of $\widetilde{L}$ acts with one character over each $V\left(\lambda_{i}\right)$; moreover $Z(\widetilde{L}) \cdot \sum_{1}^{m} v_{i}$ has dimension equal to $m$, because $\widetilde{L} \cdot \sum_{1}^{m} v_{i}$ is dense in $Z$. Thus there is $t \in Z(\widetilde{L})$ such that $t \sum v_{i}=-v_{1}+\sum_{i>1} v_{i}$, so $n t \in H_{\widetilde{L}}$. Therefore $H_{\widetilde{L}}$ is not generated by $\widetilde{L}^{\theta}$ and $H_{L_{0}}$, a contradiction.

In the rest of this section, we always suppose that $L$ is $\theta$-stable. We choose, as basis of the root system $R_{L}$ of $(L, T)$, the basis associated to the Borel subgroup $B_{L}$. Let $R_{L, \theta}$ be the restricted root system of $(L, \theta)$; it is contained in $R_{G, \theta}$. We arbitrarily choose an order of the connected components of the Dynkin diagram of $R_{L, \theta}$ and we define $R_{L, \theta}^{j}$ as the subroot system of $R_{L, \theta}$ corresponding to the $j$-th connected component of the Dynkin diagram. Let $\left\{\alpha_{1}^{j}, \ldots, \alpha_{l_{j}}^{j}\right\}$ be the set of simple roots of $R_{L, \theta}^{j}$; we index them as in [8]. Let $l$ be the rank of $G / H$ and let $n$ be the semisimple rank of $G$. In particular we suppose that, if $R_{L, \theta}^{j}$ has type $A_{l_{j}}$, then $\left(\alpha_{h}^{j}, \alpha_{k}^{j}\right) \neq 0$ only if $h$ belongs to $\{k-1, k, k+1\}$. We prove the following theorem.

Theorem 2.2. Let $X$ be a simple symmetric variety with open orbit $G / H$. Suppose that the closed orbit $Y$ is projective and that $\rho^{-1}\left(\alpha^{\vee}\right)$ is contained in $D(X)$ for each exceptional coroot $\alpha^{\vee}$ in $\rho(D(X))$. Then the closure of $T \cdot x_{0}$ in $X$ is normal. Moreover $X$ is smooth if and only if this toric variety is smooth. This is equivalent to the following conditions:

(1) The restricted root system $R_{L, \theta}$ has type $\prod_{i=1}^{p} A_{l_{i}}$ for appropriate integers $p, l_{1}, \ldots, l_{p}$. Moreover the rank of $G / H$ is greater or equal to $\sum_{i=1}^{p}\left(l_{i}+1\right)$.

(2) The cone $C(X)$ is generated by a basis of $\operatorname{Hom}_{\mathbb{Z}}(\chi(S), \mathbb{Z})$.

(3) We can index the dual basis $\left\{\lambda_{1}^{1}, \ldots, \lambda_{l_{1}+1}^{1}, \lambda_{1}^{2}, \ldots, \lambda_{l_{2}+1}^{2}, \ldots, \lambda_{l_{q}+1}^{q}\right\}$ of $\chi(S)$ so that $\left(\lambda_{h}^{j},\left(\alpha_{k}^{i}\right)^{\vee}\right)=\delta_{h, k} \delta_{i, j}$ and $\frac{1}{l_{j}+1}\left(\left(l_{j}+1\right) \lambda_{i}^{j}-i \lambda_{l_{j+1}}^{j}\right)$ is the $i$-th fundamental weight of $R_{L, \theta}^{j}$.

Before proving the theorem, we make some remarks.

Remarks 2. i) We request that $X$ is normal. Indeed there is a non normal equivariant completion $\widetilde{X}$ of a symmetric space such that the closure of $T \cdot x_{0}$ in $\widetilde{X}$ is smooth (see [18] $\S 7.2$ ). See also [16] $\S 10$ for an example of an affine nonnormal embedding $\widetilde{Z}$ of a symmetric space such that the closure of $T \cdot x_{0}$ is normal. Moreover the open orbits respectively of $\widetilde{X}$ and $\widetilde{Z}$ are not exceptional.

ii) Given $X$ which verifies the conditions of the theorem, we can almost describe the associated colored cone. The cone $C(X)$ is the dual cone of $\operatorname{cone}\left(\lambda_{1}^{1}, \ldots, \lambda_{l_{q}+1}^{q}\right)$ and $\chi(S)$ is the free abelian group with basis $\left\{\lambda_{1}^{1}, \ldots, \lambda_{l_{q}+1}^{q}\right\}$. The set $\rho(D(X))$ is constituted by the primitive vectors of the 1-faces of $C(X)$ not contained in $-C^{+}$; moreover $D(X)$ contains $\rho^{-1}\left(\left\{\alpha^{\vee}\right\}_{\alpha \in R_{L, \theta} \text { simple }}\right)$. Given any $\alpha^{\vee}$ in $\rho(D(X)) \backslash R_{L, \theta}^{\vee}$, then $\rho^{-1}\left(\alpha^{\vee}\right)$ contains two elements, while $\rho^{-1}\left(\alpha^{\vee}\right) \cap D(X)$ contains one element. 
Any of the two colors in $\rho^{-1}\left(\alpha^{\vee}\right)$ can be a color of $X$. Remark that $\rho$ is injective over $D(X)$.

iii) Suppose that $L / H_{L}$ is a group $\dot{L}$, i.e. suppose that $L=\dot{L} \times \dot{L}$ and that $\theta$ is defined by $\theta(x, y)=(y, x)$. The first condition of the theorem means that $\dot{L}$ is the quotient of a product $\Pi\left(S L_{l_{i}+1} \times \mathbb{C}^{*}\right)$ by a finite subgroup (some integer $l_{i}$ can be 0 ). If all the conditions of the theorem are verified, then $\dot{L}$ is isomorphic to the product $\Pi G L_{l_{i}+1}$ and $Z$ is isomorphic to the product $\Pi M_{l_{i}+1}$, where $M_{l_{i}+1}$ is the algebra of complex matrices of order $l_{i}+1$. Observe that the product of matrices defines a product over $\Pi M_{l_{i}+1}$ which extends the product over $\Pi G L_{l_{i}+1}$. This means that $\Pi M_{l_{i}+1}$ is an algebraic monoid whose unit group is $\Pi G L_{l_{i}+1}$.

iv) Timashev (see [16]) studied a particular class of equivariant embeddings of reductive groups. These embeddings are not necessarily normal. Here $G$ is a product $\dot{G} \times \dot{G}$ and the involution $\theta$ is defined by $\theta(x, y)=(y, x)$. Timashev supposes that there is a faithful $\dot{G}$-linear representation $V=\bigoplus V\left(\mu_{i}\right)$ with the following properties. The $\mu_{i}$ are all distinct and the map $\dot{G} \rightarrow \mathbb{P}\left(\bigoplus E n d_{\mathbb{C}}\left(V\left(\mu_{i}\right)\right)\right)$ is injective. He considers the closure of $\dot{G}$ in $\mathbb{P}\left(\bigoplus E n d_{\mathbb{C}}\left(V\left(\mu_{i}\right)\right)\right)$ and obtains a criterion for this variety to be normal, respectively smooth. Observe that $G$ acts on $\bigoplus E n d_{\mathbb{C}}\left(V\left(\mu_{i}\right)\right) \subset E n d_{\mathbb{C}}(V)$ and the action coincides with the one over $V \otimes V^{*}$ through the canonical isomorphism $\operatorname{End}_{\mathbb{C}}(V) \cong V \otimes V^{*}$. Also Renner (see [12]) considers the case where $G / H$ is isomorphic to a reductive group $\dot{G}$, but he studies the affine (normal) equivariant embeddings of $\dot{G}$ with a $G$-fixed point. He classifies the smooth ones in the case where the center of $\dot{G}$ has dimension 1 and the derived group of $\dot{G}$ is simple.

Proof. First we reduce to affine case. Observe that $X$ is smooth if and only if $Z$ is smooth. Let $U$ (respectively $U_{L}$ ) be the unipotent radical of $B$ (respectively of $\left.B_{L}\right)$, then $\mathbb{C}[Z]^{U_{L}}$ is isomorphic to $\mathbb{C}\left[X_{B}\right]^{U}$, so $\mathbb{C}(G / H)^{(B)} / \mathbb{C}^{*}$ is isomorphic to $\mathbb{C}\left(L / H_{L}\right)^{\left(B_{L}\right)} / \mathbb{C}^{*}$; in our case $T$ is contained in $L$ and we can identify both $\mathbb{C}(G / H)^{(B)} / \mathbb{C}^{*}$ and $\mathbb{C}\left(L / H_{L}\right)^{\left(B_{L}\right)} / \mathbb{C}^{*}$ with $\chi_{*}(S)$. We can identify $\mathbb{C}[X]^{(B)} / \mathbb{C}^{*}$ with $C(X)^{\vee} \cap \chi(S)$ and $\mathbb{C}[Z]^{\left(B_{L}\right)} / \mathbb{C}^{*}$ with $C(Z)^{\vee} \cap \chi(S)$ (see [3] §3.2); in particular we can identify $C(X) \subset \chi_{*}(S) \otimes \mathbb{Q}$ with $C(Z) \subset \chi_{*}(S) \otimes \mathbb{Q}$. Let $\operatorname{cl}\left(T \cdot x_{0}, X\right)$ (respectively $\overline{T \cdot x_{0}}$ ) be the closure of $T \cdot x_{0}$ in $X$ (respectively in $Z$ ). To reduce to affine case it is now sufficient to prove that $c l\left(T \cdot x_{0}, X\right)$ is normal (respectively smooth) if and only if $\overline{T \cdot x_{0}}$ is normal (respectively smooth). This fact holds because the intersection of $\operatorname{cl}\left(T \cdot x_{0}, X\right)$ with $X_{B}$ is $\overline{T \cdot x_{0}}$, so $\overline{T \cdot x_{0}}$ is open in $\operatorname{cl}\left(T \cdot x_{0}, X\right)$. Moreover $\operatorname{cl}\left(T \cdot x_{0}, X\right)$ is covered by the translates of $\overline{T \cdot x_{0}}$ with respect to the action of $N_{G^{\theta}}\left(T^{1}\right)$.

Observe that the combinatorial data $C(Z), D(Z), \chi(S)$ of $Z$ does not change if we substitute $L$ by a finite cover or by a quotient by a normal subgroup contained in $H_{L}$. Thus we can substitute $L$ with a group of simply connect type, as we have done with $G$ in the Remark 1 . The only problem is that the group obtained is no longer a subgroup of $G$. On the other hand we can restrict to consider $Z$, "forgetting" $X$. In the following, we always consider an affine embedding $Z$ of a symmetric homogeneous variety $L / H_{L}$, where $L$ is as in the Remark 1 .

Lemma 2.8. The closure of $T \cdot x_{0}$ in $Z$ is normal.

Proof of Lemma 2.8. We have a commutative diagram

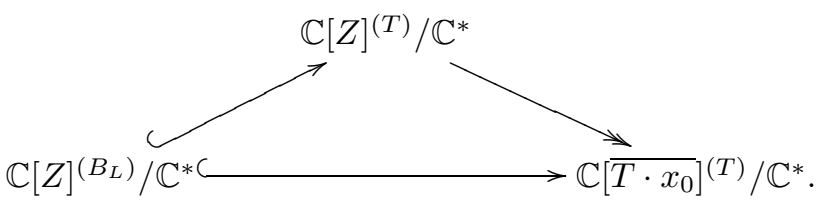


Let $W_{L, \theta}$ be the Weyl group of $R_{L, \theta}$; it is isomorphic to $N_{L^{\theta}}\left(T^{1}\right) / C_{L^{\theta}}\left(T^{1}\right)$ (see [17] Proposition 26.2). Observe that $N_{L^{\theta}}\left(T^{1}\right)$ acts on $Z$, so $W_{L, \theta}$ acts on $\mathbb{C}[Z]^{(T)} / \mathbb{C}^{*}$; moreover $N_{L^{\theta}}\left(T^{1}\right)$ acts on $\overline{T \cdot x_{0}}$, thus $W_{L, \theta}$ acts on $\mathbb{C}\left[\overline{T \cdot x_{0}}\right]^{(T)} / \mathbb{C}^{*}$. Hence the elements of $W_{L, \theta} \cdot\left(C(X)^{\vee} \cap \chi(S)\right)$ are $T$-weights in $\mathbb{C}\left[\overline{T \cdot x_{0}}\right]^{(T)} / \mathbb{C}^{*}$. To prove that $\overline{T \cdot x_{0}}$ is normal it is sufficient to show that the elements of $W_{L, \theta}$. $\left(C(X)^{\vee} \cap \chi(S)\right)$ are all the $T$-weights in $\mathbb{C}\left[\overline{T \cdot x_{0}}\right]^{(T)} / \mathbb{C}^{*}$. This holds because of the following lemma. Notice that $T \cdot x_{0}=S \cdot x_{0} \equiv S$ and $\mathbb{C}\left[\overline{T \cdot x_{0}}\right]^{(T)} / \mathbb{C}^{*} \equiv$ $\mathbb{C}\left[\overline{S \cdot x_{0}}\right]^{(S)} / \mathbb{C}^{*}$.

Lemma 2.9. Let $\lambda$ be a L-spherical weight. If there is a T-eigenvector in $\mathbb{C}[Z]$ of eigenvalue $\lambda$, then there is a $B_{L}$-eigenvector in $\mathbb{C}[Z]$ of eigenvalue $\lambda$.

Proof of Lemma 2.9. Let $v$ be a $T$-eigenvector in $\mathbb{C}[Z]$ of eigenvalue $\lambda$, we can suppose that it is contained in an irreducible $L$-subrepresentation $V(\mu)$ of $\mathbb{C}[Z]$ of highest weight $\mu$. So $\lambda=\mu-\sum a_{j} \beta_{j}$, where $a_{1}, \ldots, a_{n}$ are positive integers and $\beta_{1}, \ldots, \beta_{n}$ are the simple roots of $L$. The cone $C(Z)$ is generated by simple restricted coroots $\alpha_{1}^{\vee}, \ldots, \alpha_{r}^{\vee}$ and by appropriate vectors $v_{1}, \ldots, v_{l-r}$ in the antidominant Weyl chamber $-C^{+}$of $R_{L, \theta}$. Moreover, for each $i,\left(\lambda, v_{i}\right)=\left(\mu, v_{i}\right)+\sum a_{j}\left(\beta_{j},-v_{i}\right) \geq$ $\left(\mu, v_{i}\right) \geq 0$ because $\mu$ belongs to $C(Z)^{\vee}$. Moreover $\left(\lambda,\left(\alpha_{i}\right)^{\vee}\right) \geq 0$ for $i=1, \ldots, r$ because $\lambda$ is dominant, so $\lambda$ belongs to $C(Z)^{\vee}$. Thus there is an eigenvector in $\mathbb{C}[Z]^{\left(B_{L}\right)}$ of $T$-eigenvalue $\lambda$.

Now, we prove the necessity of the conditions.

Lemma 2.10. If $Z$ is smooth, then $[L, L]$ has type $\prod A_{i}$ and $\overline{T \cdot x_{0}}$ is smooth.

We begin considering a particular case.

Lemma 2.11. Suppose that $([L, L], \theta)$ is indecomposable and that $Z(L)$ is 1-dimensional. There are a subgroup $L^{\theta} \subset H_{L} \subset N_{L}\left(L^{\theta}\right)$ and an affine smooth embedding of $L / H_{L}$ if and only if $R_{L}$ has type $A_{l-1}$. If a such embedding exists, then it is unique, up to an equivariant isomorphism, and the closure of $T \cdot x_{0}$ in it is smooth.

Proof of Lemma 2.11. Let $\varsigma$ be the cone in $\chi_{*}(S) \otimes \mathbb{R}$ associated to $\overline{T \cdot x_{0}}$ and let $\varsigma^{\vee}$ be the dual cone. Notice that an affine embedding $Z$ of $L / H_{L}$ is smooth if and only if it is a $L$-representation; in particular $Z$ must be is an irreducible spherical representation with dimension lesser than $\operatorname{dim}[L, L]$. If moreover $[L, L] /[L, L]^{\theta}$ is isomorphic to a simple group $\dot{L}$, i.e. $[L, L]=\dot{L} \times \dot{L}$ and $\theta(x, y)=(y, x)$, then $Z$ is isomorphic, as $[L, L]$-representation, to $V(\lambda) \otimes V\left(-\omega_{0}(\lambda)\right)$. Here $\omega_{0}$ is the longest element of $W_{\dot{L}}$ and $V(\lambda)$ is a $\dot{L}$-representation with dimension lesser than $\operatorname{dim} \dot{L}$. One can easily show that there is a representation with the request properties if and only if $R_{L, \theta}$ has type $A_{l-1}$ (see the answer to ex. 24.52 in [7] p. 531 for a list of the irreducible representations of a simple $L$ with dimension at most $\operatorname{dim} L$ ). In this case $Z$ is unique up to an isomorphism of $L$-varieties. More precisely $Z$ is isomorphic as $[L, L]$-representation to $V\left(\omega_{l}\right)$ or to $V\left(\omega_{1}\right)$. Now, we do a case-tocase analysis to prove that, given a smooth $Z$, the subvariety $\overline{T \cdot x_{0}}$ is smooth. We denote by $\left\{f_{1}, \ldots, f_{n}\right\}$ the dual basis of the canonic basis $\left\{e_{1}, \ldots, e_{n}\right\}$ of $\mathbb{C}^{n}$.

i) If $([L, L], \theta)$ has type $A I$, let $Q$ be the symmetric bilinear form $\sum f_{i}^{2}$ over $\mathbb{C}^{n}$. Then the involution is defined by $\theta(A)=\left(A^{t}\right)^{-1}, L / L^{\theta}$ is $G L\left(\mathbb{C}^{n}\right) / O\left(\mathbb{C}^{n}, Q\right)$ and $Z$ is $S^{2}\left(\mathbb{C}^{n}\right)^{*}$. The subgroup $T \subset G L\left(\mathbb{C}^{n}\right)$ of diagonal matrices is a maximally split torus, the vector fixed by $L^{\theta}$ is $\sum_{i=1}^{n} f_{i}^{2}$ and $T \cdot \sum_{i=1}^{n} f_{i}^{2}$ is $\bigoplus_{i=1}^{n} \mathbb{C} f_{i}^{2}$.

ii) If $(L, L], \theta)$ has type $A I I$, let $Q$ be the antisymmetric bilinear form $\sum_{i=1}^{n} f_{i} \wedge$ $f_{i+n}$ over $\mathbb{C}^{2 n}$. Then $L / L^{\theta}$ is $G L\left(\mathbb{C}^{2 n}\right) / S p\left(\mathbb{C}^{2 n}, Q\right)$ and $Z$ is $\bigwedge^{2}\left(\mathbb{C}^{2 n}\right)^{*}$. The subgroup $T$ of diagonal matrices is a maximally split torus because it contains a maximal torus of $S p\left(\mathbb{C}^{2 n}, Q\right)$ and $\operatorname{dim} T$ is equal to the semisimple rank of $L^{\theta}$ plus the rank of $L / L^{\theta}$. The vector fixed by $L^{\theta}$ is $\sum_{i=1}^{n} f_{i} \wedge f_{i+n}$ and the closure of $T \cdot\left(\sum_{i=1}^{n} f_{i} \wedge f_{i+n}\right)$ is the vector space spanned by $f_{1} \wedge f_{n+1}, \ldots, f_{n} \wedge f_{2 n}$. 
iii) If $([L, L], \theta)$ has type $B I I$, let $Q$ be the symmetric bilinear form $\sum_{i=1}^{n} f_{i} \wedge$ $f_{i+n}+f_{2 n+1}^{2}$ over $\mathbb{C}^{2 n+1}$. Let $\varphi$ be the linear transformation of $\mathbb{C}^{2 n+1}$ such that

$$
\varphi\left(e_{i}\right)= \begin{cases}e_{n+1} & \text { if } i=1 \\ e_{1} & \text { if } i=n+1 \\ e_{i} & \text { otherwise }\end{cases}
$$

Then $L / L^{\theta}$ is $S O\left(\mathbb{C}^{2 n+1}, Q\right) \times \mathbb{C}^{*} / S O\left(\left(\bigoplus_{i \neq 1, n+1} \mathbb{C} e_{i} \oplus \mathbb{C}\left(e_{1}+e_{n+1}\right)\right), Q\right) \times\{ \pm i d\}$ and the involution over $S O\left(\mathbb{C}^{2 n+1}, Q\right)$ is the conjugation by the $\varphi$. The representation $Z$ is $\mathbb{C}^{2 n+1}$, over which $\mathbb{C}^{*}$ acts by twice a basic character. The subgroup $T$ generated by the diagonal matrices and by the center is a maximally split torus, the vector fixed by $L^{\theta}$ is $e_{1}-e_{n+1}$ and the closure of $T \cdot\left(e_{1}-e_{n+1}\right)$ is $\mathbb{C} e_{1} \oplus \mathbb{C} e_{n+1}$.

iv) If $([L, L], \theta)$ has type $D I I$, let $Q$ be the symmetric bilinear form $\sum_{i=1}^{n} f_{i} f_{i+n}$ over $\mathbb{C}^{2 n}$. Then $L / L^{\theta}$ is $S O\left(\mathbb{C}^{2 n}, Q\right)$

$\times \mathbb{C}^{*} / S O\left(\left(\bigoplus_{i \neq 1, n+1} \mathbb{C} e_{i} \oplus \mathbb{C}\left(e_{1}+e_{n+1}\right)\right), Q\right) \times\{ \pm i d\}$ and $Z$ is $\mathbb{C}^{2 n}$, over which $\mathbb{C}^{*}$ acts by twice a basic character. The subgroup $T$ generated by the diagonal matrices and by the center is a maximally split torus because $\operatorname{dim} T$ is equal to the semisimple rank of $L^{\theta}$ plus the rank of $L / L^{\theta}$. The vector fixed by $L^{\theta}$ is $e_{1}-e_{n+1}$ and the closure of $T \cdot\left(e_{1}-e_{n+1}\right)$ is the vector space spanned by $e_{1}$ and $e_{n+1}$.

v) If $([L, L], \theta)$ has type $E I V, L / L^{\theta}$ is $E_{6} \times \mathbb{C}^{*} / F_{4} \times\{ \pm i d\}$ and $Z$ is $V\left(\omega_{1}\right)$. We will reduce to the already examined case of $G L_{6} / S p_{6}$. First, we recall some facts from [9]. Let $\mathbb{A}_{\mathbb{C}}$ be either the complexification $\mathbb{Q}_{\mathbb{C}}$ of the quaternion or the complexification $\mathbb{O}_{\mathbb{C}}$ of the octonions. Given $a \in \mathbb{A}$, let $\bar{a}$ be its conjugate. Let $\mathcal{J}_{3}\left(\mathbb{A}_{\mathbb{C}}\right)$ be the space of $\mathbb{A}_{\mathbb{C}}$-Hermitian matrices of order three, with coefficients in $\mathbb{A}_{\mathbb{C}}:$

$$
\mathcal{J}_{3}\left(\mathbb{A}_{\mathbb{C}}\right)=\left\{\left(\begin{array}{lll}
r_{1} & \bar{x}_{3} & \bar{x}_{2} \\
x_{3} & r_{2} & \bar{x}_{1} \\
x_{2} & x_{1} & r_{3}
\end{array}\right), r_{i} \in \mathbb{C}, x_{i} \in \mathbb{A}_{\mathbb{C}}\right\} .
$$

$\mathcal{J}_{3}\left(\mathbb{A}_{\mathbb{C}}\right)$ has the structure of a Jordan algebra with multiplication $A \circ B:=$ $\frac{1}{2}(A B+B A)$, where $A B$ is the usual matrix multiplication. There is a well defined cubic form on $\mathcal{J}_{3}\left(\mathbb{A}_{\mathbb{C}}\right)$ called the determinant. The subgroup of $G L_{\mathbb{C}}\left(\mathcal{J}_{3}\left(\mathbb{O}_{\mathbb{C}}\right)\right)$ preserving the determinant is $E_{6}$ and contains the subgroup preserving the Jordan multiplication, namely $F_{4}$. Moreover $S L_{6}$ is contained in $E_{6} \cap G L_{\mathbb{C}}\left(\mathcal{J}_{3}\left(\mathbb{Q}_{\mathbb{C}}\right)\right)$ and $S p_{6}=S L_{6} \cap F_{4}$. There is a simple subgroup $K$ of $F_{4}$ of type $A_{1}$ such that $S L_{6} \cdot K \subset$ $E_{6}$ contains a maximal torus $T$ of $E_{6} ; T \times Z(L)^{0}$ is maximally split because the rank of $E_{6}$ is the sum of the rank of $F_{4}$ plus the rank of $E_{6} / F_{4}$. The group $F_{4}$ fixes the identity matrix because $F_{4}$ preserve the Jordan product. Notice that $\mathcal{J}_{3}\left(\mathbb{O}_{\mathbb{C}}\right)$ is the first fundamental representation of $E_{6}$, while $\mathcal{J}_{3}\left(\mathbb{Q}_{\mathbb{C}}\right)$ is the second fundamental representation of $S L_{6}$. Let $T^{1}$ be the maximal split torus contained in $T \times Z(L)^{0}$, then $T^{1}$ is contained $S L_{6} \times Z(L)^{0}, T^{1} \cdot I d$ is contained $\mathcal{J}_{3}\left(\mathbb{Q}_{\mathbb{C}}\right)$ and $S p_{6}$ fixes $I d$. (We have assumed that $Z(L)^{0} /\{ \pm 1\}$ is the connected center of $G L_{\mathbb{C}}\left(\mathcal{J}_{3}\left(\mathbb{O}_{\mathbb{C}}\right)\right.$ ).)

Proof of Lemma 2.10. We proceed in a similar manner to the proof of Theorem 2.1. Write $L=\prod L_{j} \times \widetilde{T}$, where the $L_{j}$ are $\theta$-stable semisimple normal subgroups of $L$, the $\left(L_{j}, \theta\right)$ are simple and $\widetilde{T}$ is a split central torus. Suppose that there is a smooth affine embedding $Z$ of $L / H_{L}$, for an opportune subgroup $H_{L}$, and write $Z$ as a sum of irreducible representations, say $Z=\bigoplus V\left(\lambda_{h}\right)$. Write $x_{0}=\sum v_{h}$ with $v_{h} \in V\left(\lambda_{h}\right)$, then $L \cdot v_{h}$ is dense in $V\left(\lambda_{h}\right)$. On the other hand, $\widetilde{T} \cdot v_{h}$ is contained in $\mathbb{C}^{*} v_{h}$, so $\operatorname{dim} L \cdot v_{h} \leq \operatorname{dim}[L, L] \cdot v_{h}+1$. Write $\lambda_{i}=\sum \mu_{i, j}+\nu_{i}$, where $\mu_{i, j}$ is a $L_{j}$-weight for each $j$ and $\nu_{i}$ is a $\widetilde{T}$-character; the $V\left(\mu_{i, j}\right)$ contain a $L^{\theta}$-fixed vector, in particular $\operatorname{dim} V\left(\mu_{i, j}\right) \geq \operatorname{dim} L_{j} / H_{L_{j}}+1$ for each $i$ and 
$j$. The $[L, L]$-representation $V\left(\sum_{j=1, \ldots, r} \mu_{i, j}\right)$ is the tensor product of the $L_{j^{-}}$ representations $V\left(\mu_{i, j}\right)$. Therefore, if $\mu_{i, j} \neq 0$ for at least two index $j_{1}$ and $j_{2}$, then $\operatorname{dim} V\left(\lambda_{i}\right)=\operatorname{dim} V\left(\sum \mu_{i, j}\right)=\prod_{j \mid \mu_{i, j} \neq 0} \operatorname{dim} V\left(\mu_{i, j}\right)>\sum_{j \mid \mu_{i, j} \neq 0} \operatorname{dim} V\left(\mu_{i, j}\right) \geq$ $\sum_{j \mid \mu_{i, j} \neq 0}\left(\operatorname{dim}\left(L_{j} / H_{L_{j}}\right)+1\right) \geq \operatorname{dim} L \cdot v_{i}$ (indeed $\operatorname{dim} L_{j}^{\prime} / H_{L_{j}^{\prime}} \geq 2$ for each $j$ ). Thus $V\left(\lambda_{i}\right)$ is a smooth affine equivariant embedding of $L_{j_{i}} T_{i} \cdot v_{i}$ for an appropriate index $j_{i}$ and an appropriate one-dimensional subtorus $T_{i}$ of $\widetilde{T}$; moreover $S t a b_{L_{j}}\left(v_{i}\right) \subset N_{L_{j_{i}}}\left(L_{j_{i}}^{\theta}\right)$. Every $L_{i}$ acts non-trivially over exactly one $V\left(\lambda_{i}\right)$ and the $T \cdot x_{0}$ is the product of the varieties $T \cdot v_{i}$, because $L \cdot x_{0}$ is dense in $Z$. Thus $\overline{T \cdot x_{0}}$ is the product of the closures of the $T \cdot v_{i}$ (in the $V\left(\lambda_{i}\right)$ ). By the previous lemma such closures are smooth and the $L_{i}$ have type $A_{l_{i}}$.

Lemma 2.12. If $\overline{T x_{0}}$ is smooth, then the conditions of the theorem are verified.

The hypothesis implies that $\varsigma^{\vee}$ is generated by a basis $e_{1}, \ldots, e_{l}$ of $\chi(S)$. Now, we want to describe $\mathbb{C}\left[\overline{T \cdot x_{0}}\right]$ with relation to the restricted root system of $(L, \theta)$ (when $\overline{T x_{0}}$ is smooth). Recall that the elements of $W_{L, \theta} \cdot C(X)^{\vee} \cap \chi(S)$ are the $T$ weights in $\mathbb{C}\left[\overline{T \cdot x_{0}}\right]^{(T)} / \mathbb{C}^{*}$ and that $\mathbb{C}\left[\overline{T \cdot x_{0}}\right]$ is generated by $S$-seminvariant vectors $v\left(e_{1}\right), \ldots, v\left(e_{l}\right)$ of weight respectively $e_{1}, \ldots, e_{l}$. The symmetric group $S_{l}$ acts on $\left\{e_{1}, \ldots, e_{l}\right\}$. Let $\sigma_{i, j}$ be the transposition that exchanges $e_{i}$ with $e_{j}$. The action of $W_{L, \theta}$ on $\chi(S) \otimes \mathbb{R}=\bigoplus \mathbb{R} e_{i}$ is induced by the action of $N_{L^{\theta}}\left(T^{1}\right)$ on $\mathbb{C}\left[T \cdot x_{0}\right]^{(T)}$. Moreover $N_{L^{\theta}}\left(T^{1}\right)$ stabilizes $\mathbb{C}\left[\overline{T \cdot x_{0}}\right]^{(T)}$, so $W_{L, \theta}$ stabilizes the basis $\left\{e_{1}, \ldots, e_{l}\right\}$ of $\chi(S) \otimes \mathbb{R}$; in particular $W_{L, \theta}$ exchanges the $e_{i}$. We want to describe the image of $W_{L, \theta}$ in $S_{l}$.

Lemma 2.13. If $\overline{T \cdot x_{0}}$ is smooth, then, for every restricted $L$-root $\alpha$, there are two indices $i$ and $j$ such that $\alpha=e_{i}-e_{j}$; in particular $\sigma_{\alpha}=\sigma_{i, j}$. Moreover $R_{L, \theta}$ is reduced.

Proof of the Lemma 2.13. The orthogonal reflection $\sigma_{\alpha}$ of $\chi(S) \otimes \mathbb{R}$ stabilizes the basis $\left\{e_{1}, \ldots, e_{l}\right\}$. Thus $\sigma_{\alpha}$ can exchange only two of the $e_{i}$, otherwise the $(-1)$ eigenspace would have dimension at least 2. Suppose now that $\sigma_{\alpha}=\sigma_{i, j}$, then $\sigma_{\alpha}\left(e_{i}-e_{j}\right)=-\left(e_{i}-e_{j}\right)$, so $e_{i}-e_{j}$ is a multiple of $\alpha$. Moreover it must be an integral multiple because $e_{i}-e_{j}=\sigma_{\alpha}\left(e_{j}\right)-e_{j}$ and $e_{j}$ is a weight. We can write $\alpha$ as an integral linear combination of the $e_{j}$ in a unique way, so $e_{i}-e_{j}= \pm \alpha$. Moreover, there is not a restricted root $\alpha$ such also $2 \alpha$ is a restricted root. Indeed, by the previous part of the proof, there is $i$ and $j$ such that $\sigma_{\alpha}=\sigma_{2 \alpha}=\sigma_{i, j}$ and $\alpha=2 \alpha= \pm\left(e_{i}-e_{j}\right)$, a contradiction.

Lemma 2.14. If $\overline{T \cdot x_{0}}$ is smooth, then, for each fixed $j$, there is a subset $\left\{e_{1}^{j}, \ldots, e_{l_{j}+1}^{j}\right\}$ of $\left\{e_{1}, \ldots, e_{l}\right\}$ such that the simple restricted roots in $R_{L, \theta}^{j}$ are $e_{1}^{j}-e_{2}^{j}, \ldots, e_{l_{j}}^{j}-e_{l_{j}+1}^{j}$; in particular $R_{L, \theta}^{j}$ has type $A_{l_{j}}$.

Proof of the Lemma 2.14. The vectors $e_{i}-e_{j}$ and $e_{h}-e_{k}$ are orthogonal if and only if the sets $\{i, j\}$ and $\{h, k\}$ are disjoint. Indeed $\frac{2\left(e_{h}-e_{k}, e_{i}-e_{j}\right)}{\left(e_{i}-e_{j}, e_{i}-e_{j}\right)}\left(e_{i}-e_{j}\right)=$ $\left(e_{h}-e_{k}\right)-\sigma_{e_{i}-e_{j}}\left(e_{h}-e_{k}\right)$. Clearly we can choose $e_{1}^{j}$ and $e_{2}^{j}$ so that $\alpha_{1}^{j}$ is equal to $e_{1}^{j}-e_{2}^{j}$. Suppose that there is a subset $\left\{e_{1}^{j}, \ldots, e_{s}^{j}\right\}$ such that $\alpha_{i}^{j}$ is equal to $e_{i}^{j}-e_{i+1}^{j}$ for each $i<s$ (with $s<l_{j}+1$ ) and let $e_{h}-e_{k}$ be a simple root $\alpha_{s}^{j}$ which is not orthogonal to the space generated by $\alpha_{1}^{j}, \ldots, \alpha_{s-1}^{j}$. Neither $e_{h}$ nor $e_{k}$ belongs to $\left\{e_{1}^{j}, \ldots, e_{s-1}^{j}\right\}$, otherwise the Dynkin diagram would contain a cycle; thus $\left(\alpha_{s}^{j}, \alpha_{i}^{j}\right)=0$ for each $i$ strictly lesser than $s-1$. Hence $\left(\alpha_{s}^{j}, \alpha_{s-1}^{j}\right) \neq 0$, so $e_{s}^{j}$ is equal either to $e_{h}$ or to $e_{k}$. On the other hand, $e_{s}^{j}$ cannot be equal to $e_{k}$, otherwise the root $\sigma_{\alpha_{s}^{j}}\left(\alpha_{s-1}^{j}\right)$ is equal to $e_{s-1}^{j}-e_{h}=e_{s-1}^{j}-e_{k}+e_{k}-e_{h}=\alpha_{s-1}^{j}-\alpha_{s}^{j}$. Thus $e_{s}^{j}=e_{h}$, we can define $e_{s+1}^{j}=e_{k}$ and the first part of the lemma is proved by 
induction. In particular, the Weyl group of $R_{L, \theta}^{j}$ is isomorphic to $S_{l_{j}+1}$. Therefore $R_{L, \theta}^{j}$ has type $A_{l_{j}}$, because $R_{L, \theta}$ is reduced by the Lemma 2.13.

Remark 3. The sets $\left\{e_{1}^{j}, \ldots, e_{l_{j}+1}^{j}\right\}$ are pairwise disjoint because the sets $R_{L, \theta}^{j}$ are pairwise orthogonal. Thus we can index the basis $\left\{e_{1}, \ldots, e_{l}\right\}$ as $\left\{e_{1}^{1}, \ldots, e_{l_{1}+1}^{1}, e_{1}^{2}, \ldots\right.$, $\left.e_{l_{2}+1}^{2}, \ldots, e_{l_{q}+1}^{q}\right\}$, so that $\alpha_{i}^{j}=e_{i}^{j}-e_{i+1}^{j}$ for all $i$ and $j$.

Lemma 2.15. Suppose $\overline{T \cdot x_{0}}$ smooth and let $\lambda_{i}^{j}$ be $\sum_{h=1}^{i} e_{h}^{j}$ for each $i$ and $j$. Then $C(X)^{\vee} \cap \chi(S)$ is generated by the $\lambda_{i}^{j}$.

Proof of the Lemma 2.15. By the Lemma 2.9 and the diagram (**) we have $C(X)^{\vee}=$ cone $\left(e_{1}^{1}, \ldots, e_{l_{q}+1}^{q}\right) \cap C^{+}$. Using the Lemma 2.13 , one can easily show that the $i$-th fundamental weight $\omega_{i}^{j}$ of $R_{L, \theta}^{j}$ is equal to $\frac{1}{l_{j}+1}\left(\left(l_{j}+1\right) \lambda_{i}^{j}-i \lambda_{l_{j+1}}^{j}\right)$. Moreover, $\lambda_{l_{j}+1}^{j}$ is invariant by $W_{L, \theta}$ for each $j$, so it is orthogonal to $\operatorname{span}_{\mathbb{R}}\left(R_{L, \theta}\right)$. Hence cone $\left(e_{1}^{1}, \ldots, e_{l_{q}+1}^{q}\right) \cap C^{+}$is equal to $\operatorname{cone}\left(\lambda_{1}^{1}, \ldots, \lambda_{l_{q}+1}^{q}\right)$. Indeed the restriction of $\left(\omega_{i}^{j}, \cdot\right)$ to $\operatorname{span}_{\mathbb{R}}\left(R_{L, \theta}\right)$ coincides with the restriction of $\left(\lambda_{i}^{j}, \cdot\right)$ for each $i \leq l_{j}$. On the other hand, $C^{+} \subset$ cone $\left(e_{1}^{1}, \ldots, e_{l_{q}+1}^{q}\right)+\operatorname{cone}\left(-e_{l_{1}+1}^{1},-e_{l_{2}+1}^{2}, \ldots,-e_{l_{q}+1}^{q}\right)$; moreover, if a vector $\sum a_{i}^{j} \lambda_{i}^{j}=\sum b_{i}^{j} e_{i}^{j}$ belongs to cone $\left(e_{1}^{1}, \ldots, e_{l_{q}+1}^{q}\right)$, then $a_{l_{j}+1}^{j}=b_{l_{j}+1}^{j}$ is positive for each $j$.

Conclusion of the proof of the Lemma 2.12. The Lemma 2.15 implies the second condition of Theorem 2.2. The type of $R_{L, \theta}$ is $\prod_{i=1}^{p} A_{l_{i}}$ by the Lemma 2.14; on the other hand $\operatorname{rank} G / H \geq \sum_{i=1}^{p}\left(l_{i}+1\right)$ because of the Lemma 2.13 and of the Remark 3. The last condition of Theorem 2.2 is easily verified using the Lemma 2.13 and the definition of the $\lambda_{i}^{j}$.

Next, we come to the converse of Lemma 2.12.

Lemma 2.16. Suppose that the conditions of the Theorem 2.2 are verified. For all $j$, let $e_{1}^{j}$ be $\lambda_{1}^{j}$ and let $e_{i+1}^{j}=\sigma_{\alpha_{i}^{j}} e_{i}^{j}$ for every $i \leq l_{j}$. Then $\varsigma^{\vee} \cap \chi(S)$ is $\bigoplus_{i, j} \mathbb{Z}^{+} e_{i}^{j}$ and $e_{i+1}^{j}=\lambda_{i+1}^{j}-\lambda_{i}^{j}$ for each $1 \leq i \leq l_{j}$; in particular $\overline{T \cdot x_{0}}$ is smooth.

Proof of the Lemma 2.16. Recall that $\overline{T \cdot x_{0}}$ is the toric variety associated to the cone $\varsigma^{\vee}=W_{L, \theta} \cdot C(Z)^{\vee}$, where $C(Z)^{\vee}$ is equal to cone $\left(\lambda_{1}^{1}, \ldots, \lambda_{l_{q}+1}^{q}\right)$; thus cone $\left(e_{1}^{1}, \ldots, e_{l_{q}+1}^{q}\right)$ is contained in $\varsigma^{\vee}$. We define $\omega_{0}^{j}:=\lambda_{0}^{j}:=0$ for all $j$ (we use such definition only in the current proof). We prove that $e_{i+1}^{j}=\lambda_{i+1}^{j}-\lambda_{i}^{j}$ by induction on $i$. Suppose that this holds for $i-1$ and notice that $\lambda_{h}^{j}=\omega_{h}^{j}+\frac{h}{l_{j}+1} \lambda_{l_{j}+1}^{j}$ for each $j$ and $h<l_{j}+1$ (here $\omega_{h}^{j}$ is the $h$-th fundamental weight of $R_{L, \theta}^{j}$ ). We have $e_{i+1}^{j}=\sigma_{\alpha_{i}^{j}}\left(\lambda_{i}^{j}-\right.$ $\left.\lambda_{i-1}^{j}\right)=\frac{1}{l_{j}+1} \lambda_{l_{j}+1}^{j}+\sigma_{\alpha_{i}^{j}}\left(\omega_{i}^{j}-\omega_{i-1}^{j}\right)=\frac{1}{l_{j}+1} \lambda_{l_{j}+1}^{j}+\omega_{i+1}^{j}-\omega_{i}^{j}=\lambda_{i+1}^{j}-\lambda_{i}^{j}$, because the $\lambda_{l_{j}+1}^{j}$ are invariant under $W_{L, \theta}$. The equality $e_{i+1}^{j}=\frac{1}{l_{j}+1} \lambda_{l_{j}+1}^{j}+\omega_{i+1}^{j}-\omega_{i}^{j}$ implies that $\sigma_{\alpha_{h}^{j}} e_{i+1}^{j} \neq e_{i+1}^{j}$ only if $h$ is equal to $i+1$ or to $i$. Thus cone $\left(e_{1}^{1}, \ldots, e_{l_{q}+1}^{q}\right)$ is stabilized by $W_{L, \theta}$, hence it is equal to $\varsigma^{\vee}$ because it contains $C(Z)^{\vee}$.

Remark 4. Suppose that the conditions of the Theorem 2.2 are verified. The previous lemma together with the Lemma 2.13 imply that $\alpha_{1}^{j}=2 \lambda_{1}^{j}-\lambda_{2}^{j}$ and $\alpha_{i}^{j}=-\lambda_{i-1}^{j}+2 \lambda_{i}^{j}-\lambda_{i+1}^{j}$ for each $j$ and $i>1$.

In the following lemma we need that $L$ is as in the Remark 1; indeed, without such assumption the Lemma 2.18 is false. We will use also the Lemma 2.12.

Lemma 2.17. If $\overline{T \cdot x_{0}}$ is smooth, then $Z$ is smooth.

First, we consider a particular case. Recall that, supposed $\overline{T \cdot x_{0}}$ smooth, $R_{L, \theta}$ has type $\prod A_{l_{j}}$. 
Lemma 2.18. Suppose that $R_{L, \theta}$ has type $A_{l-1}$ and that $\operatorname{dim} Z(L)=1$. Then, there is one subgroup $L^{\theta} \subset H_{L} \subset N_{L}\left(L^{\theta}\right)$ and one affine embedding $Z$ of $L / H_{L}$ such that the closure of $T \cdot x_{0}$ in $Z$ is smooth (we request that $H_{L} \cap Z(L)^{0} \equiv$ $\left.\{ \pm 1\} \subset \mathbb{C}^{*} \equiv Z(L)^{0}\right)$. Moreover: i) $H_{L}$ and $Z$ are unique up to an equivariant isomorphism; ii) $Z$ is smooth.

Proof of the Lemma 2.18. We have already show in the proof of the Lemma 2.11 that there are one subgroup $H^{\prime}$ and one affine $L$-variety $Z^{\prime}$ such that: 1) $L^{\theta} \subset H^{\prime} \subset$ $N_{L}\left(L^{\theta}\right)$; 2) $Z^{\prime}$ is an affine smooth embedding of $L / H^{\prime}$; such variety is unique up to an equivariant isomorphism and the closure of $T \cdot x_{0}$ in it is smooth. Therefore, by the Lemma 2.16, it is sufficient to prove that there is one $L^{\theta} \subset H_{L} \subset N_{L}\left(L^{\theta}\right)$ and one affine embedding of $L / H_{L}$ (up to equivariant isomorphism) satisfying the conditions of the Theorem 2.2.

Let $H_{L}$ be a subgroup of $L$ and let $Z$ be an embedding of $L / H_{L}$ satisfying the conditions of the Theorem 2.2; write $C(Z)=\operatorname{cone}\left(\left(\alpha_{1}^{1}\right)^{\vee}, \ldots,\left(\alpha_{l-1}^{1}\right)^{\vee}, v_{1}\right)$. To determine $H_{L}$ is sufficient to determine $H_{T}$ (see [18] $\S 2.2$ Lemme 2). First, we determine the subgroup $H_{T \cap[L, L]}$. Observe that $\bigoplus_{i=1}^{l-1} \mathbb{Z}\left(\alpha_{i}^{1}\right)^{\vee}=\chi_{*}(T \cap[L, L] / T \cap$ $\left.[L, L]^{\theta}\right)$ has finite index in $\chi_{*}\left(T \cap[L, L] / H_{T \cap[L, L]}\right)$ and that $\chi_{*}\left(T \cap[L, L] / H_{T \cap[L, L]}\right)$ is contained in $\chi_{*}(S)$. Hence $\chi_{*}\left(T \cap[L, L] / H_{T \cap[L, L]}\right)$ is $\bigoplus_{i=1}^{l-1} \mathbb{Z}\left(\alpha_{i}^{1}\right)^{\vee}$ and $H_{T \cap[L, L]}$ is $T \cap[L, L]^{\theta}$. On the other hand we have supposed that $H_{Z(L)^{0}}=\left(Z(L)^{0}\right)^{\theta}$, thus $\chi:=\chi\left(T \cap[L, L] / H_{T \cap[L, L]} \times Z(L)^{0} / H_{Z(L)^{0}}\right)$ is $\chi\left(T / T^{\theta}\right)=\bigoplus_{i=1}^{l-1} \mathbb{Z} \omega_{i}^{1} \oplus \mathbb{Z} \lambda_{l}^{1}$, so $\chi(S)$ has index $l$ in $\chi$ (observe that $\chi\left(Z(L)^{0}\right) \otimes \mathbb{R}=\mathbb{R} \lambda_{l}^{1}$ because $\lambda_{l}^{1}$ is orthogonal to all the simple restricted coroots). There is exactly one subgroup $K$ of $N_{L}\left(L^{\theta}\right) / L^{\theta}$ of order $l$ which does not intersect neither $N_{[L, L]}\left([L, L]^{\theta}\right) /[L, L]^{\theta}$ nor $Z(L)^{0} /\{ \pm i d\}$. Indeed notice that $N_{[L, L]}\left([L, L]^{\theta}\right) /[L, L]^{\theta}$ is isomorphic to the fundamental group of $R_{L, \theta}$, namely the ciclic group of order $l$; on the other hand the unique subgroup of $\mathbb{C}^{*}$ of order $l$ is the group of $l$-th roots of the unity. Therefore $H_{L} / L^{\theta}$ is equal to $K$, so $\chi_{*}(S)$ and $H_{L}$ are univocally determined. We can suppose, by the Remark 4 , that $\left(\alpha_{i}^{1}, v_{1}\right)=-\delta_{i, l-1}$, up to an isomorphism of the Dynkin diagram. On the other hand, $\left\{\left(\alpha_{1}^{1}\right)^{\vee}, \ldots,\left(\alpha_{l-1}^{1}\right)^{\vee}, v_{1}\right\}$ is a basis of $\chi_{*}(S)$, thus the image of $v_{1}$ by the projection $\left(\chi_{*}\left(Z(L)^{0}\right) \otimes \mathbb{R}\right) \oplus \bigoplus \mathbb{R}\left(\alpha_{i}^{1}\right)^{\vee} \rightarrow \chi_{*}\left(Z(L)^{0}\right) \otimes \mathbb{R}$ is determined up the sign. Therefore $C(Z)$ is univocally determined up the automorphism of $L$ which is the identity on $[L, L]$ and coincides with $\theta$ on $Z(L)^{0}$. On the other hand, $D(Z)=D\left(L / H_{L}\right)$ because $Z$ is affine and $\operatorname{dim} C(Z)=\operatorname{rank} \chi_{*}(S)$ (see [3] Corollaire 2, page 51). Therefore $Z$ is univocally determined, up to automorphism.

Proof of the Lemma 2.17. Let $\left\{\left(\alpha_{1}^{1}\right)^{\vee}, \ldots,\left(\alpha_{l_{1}}^{1}\right)^{\vee}, v_{1},\left(\alpha_{1}^{2}\right)^{\vee}, \ldots,\left(\alpha_{l_{2}}^{2}\right)^{\vee}, v_{2}, \ldots, v_{q}\right\}$ be the dual basis of $\left\{\lambda_{1}^{1}, \ldots, \lambda_{l_{1}+1}^{1}, \lambda_{1}^{2}, \ldots, \lambda_{l_{2}+1}^{2}, \ldots, \lambda_{l_{q}+1}^{q}\right\}$. Write $L=\prod L_{i} \times \widetilde{T}$ with $\left(L_{i}, \theta\right)$ indecomposable and $\widetilde{T}=Z(L)^{0}$. Let $T_{i}$ be the inverse image in $T^{1}$ of $\operatorname{Im}\left(v_{i}\right) \subset S$ and define $L_{i}^{\prime}=L_{i} T_{i}$. Observe that $\chi_{*}\left(Z(L)^{0}\right)$ is $\frac{1}{2} \chi_{*}\left(Z(L)^{0} / H_{Z(L)^{0}}\right)$, $\chi_{*}\left(T^{1} \cap L_{i}^{\prime} / H_{T^{1} \cap L_{i}^{\prime}}\right)$ is $\mathbb{Z} v_{i} \oplus \bigoplus_{j=1}^{l_{j}+1} \mathbb{Z}\left(\alpha_{j}^{i}\right)^{\vee}$ and $\chi_{*}(S)$ is $\bigoplus_{i} \chi_{*}\left(T^{1} \cap L_{i}^{\prime} / H_{T^{1} \cap L_{i}^{\prime}}\right)$. Thus $L$ is the direct product of the $L_{i}^{\prime}$ and $H_{L}=\prod H_{L_{i}^{\prime}}$. Hence $L / H_{L}=\prod L_{i}^{\prime} / H_{L_{i}^{\prime}}$. We have $C(Z)^{\vee}=\sum_{i=1}^{q} \operatorname{cone}\left(\lambda_{1}^{i}, \ldots, \lambda_{l_{i}+1}^{i}\right)$, hence $Z$ is the product of the affine embeddings $Z_{i}$ of $L_{i}^{\prime} / H_{L_{i}^{\prime}}$ corresponding respectively to the colored cones $\left.\left(\operatorname{cone}\left(\lambda_{1}^{i}, \ldots, \lambda_{l_{i}+1}^{i}\right)\right)^{\vee}, \rho^{-1}\left(\left\{\alpha_{1}^{\vee}, \ldots, \alpha_{l_{i}}^{\vee}\right\}\right)\right)$ These embeddings are smooth by the previous lemma, so $Z$ is smooth.

Remark 5. Let $X$ be a smooth simple symmetric variety and suppose for simplicity that $\chi(S)$ has rank equal to the rank of $R_{L}$ plus 1 , in particular that $R_{L}$ is irreducible. We want to remark that there is only one way to index the basis of $\chi(S)$ that generates $C(X)^{\vee}$ so that it verifies the third condition. First observe that indexing this basis is equivalent to indexing the dual basis of $\chi_{*}(S)$. Write $C(X) \cap \chi_{*}(S)=\bigoplus \mathbb{Z}^{+}\left(\alpha_{i}^{1}\right)^{\vee} \oplus \mathbb{Z}^{+} v_{1}$. If we request that $\left(\alpha_{i}^{1}, \alpha_{j}^{1}\right) \neq 0$ only 
if $i \in\{j-1, j, j+1\}$, then the index of $\left\{\left(\alpha_{1}^{1}\right)^{\vee}, \ldots,\left(\alpha_{l-1}^{1}\right)^{\vee}\right\}$ is defined up the non trivial automorphism of the Dynkin diagram. The third condition of the theorem implies that $\alpha_{1}^{1}=2 \lambda_{1}^{1}-\lambda_{2}^{1}$ and $\alpha_{i}^{1}=-\lambda_{i-1}^{1}+2 \lambda_{i}^{1}-\lambda_{i+1}^{1}$ for each $i>1$; in particular $\left(\alpha_{i}^{1}, v_{1}\right)=-\delta_{l-1, i}$. Hence the condition $\left(\alpha_{l-1}^{1}, v_{1}\right)=-1$ let us determine completely the indexing of the basis $\left\{\left(\alpha_{1}^{1}\right)^{\vee}, \ldots,\left(\alpha_{l-1}^{1}\right)^{\vee}, v\right\}$.

\section{Smooth complete symmetric varieties with PicARd Number one}

In this section we always suppose $G$ semisimple. We classify the smooth complete symmetric varieties with Picard number one and we prove that they are all projective. Let $X$ be a smooth complete symmetric variety of rank $l$. Let $n$ be the number of colors that do not belong to $X$ and let $m$ be the number of onedimensional cones which are faces of a colored cone belonging to the colored fan of $X$. Observe that we consider also the one-dimensional faces which do not intersect the valuation cone. It is known that the Picard group of $X$ is free and its rank is equal to $n+m-l$. In fact, the rank of $\operatorname{Pic}(X)$ is equal to cardinality of $D(G / H) \backslash D(X)$ minus the rank of $G / H$ plus the rank of the group composed by the functions over $C(X)$ which are linear over each colored cone of $X$ and assume integral value over $\chi_{*}(S) \cap C(X)$ (see [3] $\S 5.2$ Théorème); moreover the maximal colored cone are generated by a basis of $\chi_{*}(S)$. If $P i c(X)$ has rank one, then there is at most one color which does not belong to $X$. We have two cases: 1$)$ there is one color that does not belong to $X$ and $X$ is simple $(n=1$ and $m=l) ; 2)$ all the colors belong to $X$ and $m=l+1$.

Lemma 3.1. If $G / H$ has a smooth completion $X$ with Picard number one, then $G / H$ has exactly $l$ colors. Moreover, any maximal simple open $G$-stable subvariety of $X$ has still Picard number one.

Proof. There are exactly $l+1 B$-stable divisor on $X$ because $n+m=l+1$ and $\rho$ is injective over $D(X)$. Among them, there must be at least one $G$-stable divisor. (The cones spanned only by colors intersect the valuation cone just in 0 ). On the other hand, there are at least $l$ colors. Hence there are exactly $l$ colors corresponding to $l$ distinct restricted coroots.

Notice that $N(X)$ contains one vector, say $v$. Let $\tilde{X}$ be a maximal simple open $G$-subvariety of $X$. In the following we denote by $L$ the standard Levi subgroup of the stabilizer of $\widetilde{X}_{B}$. First of all, we prove that either $R_{G, \theta}$ is irreducible or has type $A_{1} \times A_{1}$; next we will do a case-by-case analysis. In some cases we will classify first the possibilities for $\widetilde{X}$ and in a second time the possibilities for $X$. Observe that in the previous section $X$ was always a simple variety.

Lemma 3.2. (1) The root system $R_{L, \theta}$ is irreducible and has type $A_{l-1}$.

(2) Denote by $\alpha$ the simple restricted root such that $\alpha^{\vee}$ does not belong to $\rho(D(\tilde{X}))$. Then $\alpha$ must be an endpoint of the Dynkin diagram of $R_{G, \theta}$.

Proof. The rank of $R_{G, \theta}$ is equal to rank of $R_{L, \theta}$ plus 1 , because $\rho(D(\widetilde{X}))$ contains $l-1$ elements and $\rho$ is injective. On the other hand, as $\widetilde{X}$ is smooth, the $\operatorname{rank}\left(R_{G, \theta}\right)$ is also greater (or equal) than $\operatorname{rank}\left(R_{L, \theta}\right)$ plus the number of connected components of the Dynkin diagram of $R_{L, \theta}$. Thus the first point of the lemma follows. To prove the second point observe that the Dynkin diagram of $R_{L, \theta}$ is obtained from the one of $R_{G, \theta}$ by removing the vertex corresponding to $\alpha$.

Lemma 3.3. The root system $R_{G, \theta}$ is irreducible or its type is $A_{1} \times A_{1}$.

Proof. Suppose that $R_{G, \theta}$ is reducible, then its type is either $A_{l-1} \times A_{1}$ or $A_{l-1} \times B C_{1}$, because of Lemma 3.2. We index the simple roots of $R_{G, \theta}$ so that 
$\alpha_{l}$ is orthogonal to the other simple roots and we denote by $\omega_{1}$ the first fundamental weight of the subroot system of type $A_{l-1}$; in particular we assume $D(\tilde{X})=\left\{D_{\alpha_{i}^{\vee}}\right\}_{i<l}$. The variety $\tilde{X}$ cannot be complete, otherwise $C(\tilde{X})$ contains $-\omega_{1}$. Notice that $C(\tilde{X})$ contains also $\omega_{1}$, because $\omega_{1}$ is a linear combination of $\alpha_{1}^{\vee}, \ldots, \alpha_{l-1}^{\vee}$ with positive coefficients. There is another maximal simple subvariety with colors $\left\{D_{\alpha_{j}^{\vee}}\right\}_{j \neq i}$ for an appropriate $i<l$, because $\widetilde{X}$ is not complete and $X$ contains exactly one $G$-stable divisor. Hence, by the Lemma 3.2 , there is $i<l$ such that $\alpha_{1}, \ldots, \widehat{\alpha}_{i}, \ldots, \alpha_{l}$ generate a root system of type $A_{l-1}$. This is possible only if $l$ is equal to 2 and $R_{G, \theta}$ has type $A_{1} \times A_{1}$.

Remarks 6 . i) by the previous two Lemmas, the variety $X$ may be non-simple only if $R_{G, \theta}$ has type $A_{1} \times A_{1}, A_{l}, B_{2}, D_{l}$ or $G_{2}$; ii) because of the Remark 5 , we know that $\left(\alpha_{i}^{\vee}, v\right)$ is equal either to $-\delta_{i, 1}$ or to $-\delta_{i, l-1}$ for each $i$ such that $\alpha_{i}^{\vee} \in \rho(D(\tilde{X}))$; iii) if $G / H$ is Hermitian then, by the Lemma 3.1, $H=N_{G}\left(G^{\theta}\right)$ and there are no exceptional roots.

In the following we do a case-by-case analysis.

1) Suppose that $R_{G, \theta}$ is reducible. We have seen that it has type $A_{1} \times A_{1}$ and that $X$ is not simple. Thus $X$ is covered by two simple open subvarieties whose associated colored cones are respectively $\left(\operatorname{cone}\left(\alpha_{1}^{\vee}, v\right),\left\{D_{\alpha_{1}^{\vee}}\right\}\right)$ and $\left(\operatorname{cone}\left(\alpha_{2}^{\vee}, v\right),\left\{D_{\alpha_{2}^{\vee}}\right\}\right)$; so $v=-\omega_{1}^{\vee}-\omega_{2}^{\vee}$ because of the Remarks 6 . Observe that the lattice generated by $\alpha_{1}^{\vee}$ and $v$ is equal to the lattice generated by $\alpha_{2}^{\vee}$ and $v$; more precisely it is the lattice $\mathbb{Z} 2 \omega_{1}^{\vee} \oplus \mathbb{Z}\left(\omega_{1}^{\vee}+\omega_{2}^{\vee}\right)$. Let $\left\{\lambda_{1}, \lambda_{2}\right\}$ be the dual basis of $\left\{\alpha_{1}^{\vee}, v\right\}$. We have $\lambda_{1}=\frac{1}{2} \alpha_{1}-\frac{1}{2} \alpha_{2}, \lambda_{2}=-\alpha_{2}$ and $\frac{1}{2}\left(2 \lambda_{1}-\lambda_{2}\right)=\frac{1}{2} \alpha_{1}=\omega_{1}$. We can proceed in the same manner for the dual basis of $\left\{\alpha_{2}^{\vee}, v\right\}$. We have proved that in this case $X$ is smooth.

In the following we always assume $R_{G, \theta}$ irreducible. Moreover, we number the simple restricted root as in [8].

Lemma 3.4. Let $(G, \theta)$ be an indecombosable involution and let (cone $\left(\alpha_{1}^{\vee}, \ldots\right.$, $\left.\left.\alpha_{l-1}^{\vee}, v\right), \rho^{-1}\left(\left\{\alpha_{1}^{\vee}, \ldots, \alpha_{l-1}^{\vee}\right\}\right)\right)$ be a colored cone in $\chi_{*}\left(T^{1}\right) \otimes \mathbb{R}$. There is at most a subgroup $G^{\theta} \subset H \subset N_{G}\left(G^{\theta}\right)$ such that the embedding $\tilde{X}$ of $G / H$ corresponding to the previous colored cone is smooth.

Proof of Lemma 3.4. Suppose that a such group exists; in particular $R_{L, \theta}$ has type $A_{l-1}$. We can suppose that $\left\{\alpha_{1}^{\vee}, \ldots, \alpha_{l-1}^{\vee}, v\right\}$ is a basis of $\chi_{*}(S)$; let $\left\{\lambda_{1}, \ldots, \lambda_{l}\right\}$ be the dual basis. We have to prove that $\chi_{*}(S)$, or equivalently $v$, is univocally determined. Notice that $v$ is determined up to a multiplicative constant because it generates a 1-dimensional face of the fixed colored cone. Moreover, if we substitute $v$ with a multiple, $\lambda_{1}, \ldots, \lambda_{l-1}$ do not change because $\left(\lambda_{i}, v\right)=0$ for each $i<l$. Thus it is sufficient to prove that there is a unique possibility for $\lambda_{l}$. By the Theorem $2.2, \lambda_{l}$ is equal to $l\left(\lambda_{1}+\widetilde{\omega}_{1}\right)$, thus it is univocally determined (here $\widetilde{\omega}_{1}$ is the first fundamental weight of the root system generated by $\left.\alpha_{1}, \ldots, \alpha_{l-1}\right)$.

2) Suppose that $R_{G, \theta}$ has rank 1. Then, for each $H$ such that $G^{\theta} \subset H \subset N_{G}\left(G^{\theta}\right)$, there is a unique (non trivial) embedding and it is simple, projective, smooth, with Picard number at most 2 and $D(X)$ equal to the empty set. The group $\operatorname{Pic}(X)$ has rank 2 if and only if $G / H$ is Hermitian and $H=G^{\theta}$. In this case we have two possibilities: 1) $(G, \theta)$ has type $A I, n=l=1, G / H$ is isomorphic to $S L_{2} / S O_{2}$, the restricted root system has type $A_{1}$ and $X$ is isomorphic to $\left.\mathbb{P}^{1} \times \mathbb{P}^{1} ; 2\right)(G, \theta)$ has type $A I V, G / H$ is isomorphic to $S L_{n+1} / S\left(L_{1} \times L_{n}\right)$, the restricted root system has type $B C_{1}, X$ is exceptional and isomorphic to $\mathbb{P}^{n} \times\left(\mathbb{P}^{n}\right)^{*}$.

In the following we always assume that $R_{G, \theta}$ has rank at least 2. We will work with the standard inner product over $\mathbb{R}^{n}$, for various $n$, and we will denote by $\left\{e_{1}, \ldots, e_{n}\right\}$ the usual orthonormal basis. One can realize a root system of type $A_{l-1}$ as the set $\left\{e_{i}-e_{j} \mid i \neq j\right\}$ contained in the vector space $\left\{\sum a_{i} e_{i} \in \mathbb{R}^{l} \mid \sum a_{i}=\right.$ 
$0\}$. Moreover, $\left\{e_{1}-e_{2}, \ldots, e_{l-1}-e_{l}\right\}$ is a basis of the root system and the $j$-th fundamental weight is $\frac{1}{l}\left(l \sum_{i=1}^{j} e_{i}-j \sum_{i=1}^{l} e_{i}\right)$.

3) Suppose that $R_{G, \theta}$ has type $A_{l}$. As before, we can realize $R_{G, \theta}$ as the set $\left\{e_{i}-e_{j} \mid i \neq j\right\} \subset \mathbb{R}^{l+1}$; in particular we can identify the restricted roots with the corresponding coroots. Up to an automorphism of the Dynkin diagram, we can suppose that $\rho(D(\widetilde{X}))=\left\{\alpha_{1}, \ldots, \alpha_{l-1}\right\}$. First, we consider the case where $X$ is simple, i.e. $\widetilde{X}$ is complete and coincides with $X$. In this case $C(\widetilde{X})$ is generated by $-\omega_{1}$ and $\rho(D(\tilde{X}))$. Indeed $-\omega_{2}=\alpha_{1}-2 \omega_{1}$ and $-\omega_{j+1}=\sum_{i=1}^{j} \alpha_{j}-\omega_{1}-$ $\omega_{j}$ for each $j=2, \ldots, l-1$. We set $f_{i}=-e_{l-i+1}$ and $\gamma_{i}=\alpha_{l-i}$, so that $\gamma_{i}=$ $f_{i}-f_{i+1}$ and $-\omega_{1}=\frac{1}{l+1}\left((l+1) f_{l}-\sum_{i=0}^{l} f_{i}\right.$ ) (we allow $i$ to be 0 ). We have renamed the simple restricted roots so that $\left(\gamma_{l-1},-\omega_{1}\right)=-1$. Let $\left\{\lambda_{1}, \ldots, \lambda_{l}\right\}$ be the dual basis of $\left\{\gamma_{1}, \ldots, \gamma_{l-1},-\omega_{1}\right\}$. We have $\lambda_{j}=\left(\sum_{i=1}^{j} f_{i}\right)-j f_{0}$, so $(1 / l)\left(l \lambda_{j}-\right.$ $\left.j \lambda_{l}\right)=(1 / l)\left(l \sum_{i=1}^{j} f_{i}-j \sum_{i=1}^{l} f_{i}\right)$ is the $j$-th fundamental weight $\widetilde{\omega}_{j}$ of the root system generated by $\gamma_{1}, \ldots, \gamma_{l-1}$. Hence, the symmetric variety with $\rho(D(\tilde{X}))=$ $\left\{\alpha_{1}, \ldots, \alpha_{l-1}\right\}, N(\tilde{X})=\left\{-\omega_{1}\right\}$ and $\chi_{*}(S)$ equal to the weight lattice of $R_{G, \theta}^{\vee}$ is smooth. It is the only possibility for a simple $X$ because of the Lemma 3.4.

Now we classify the $X$ which are not simple. In this case $X$ has two closed orbits; indeed the maximal colored cones of the colored fan of $X$ are $\left(\operatorname{cone}\left(\alpha_{1}, \ldots, \alpha_{l-1}, v\right),\left\{D_{\alpha_{1}^{\vee}}, \ldots\right.\right.$, $\left.\left.D_{\alpha_{l-1}^{\vee}}\right\}\right)$ and $\left(\right.$ cone $\left.\left(\alpha_{2}, \ldots, \alpha_{l}, v\right),\left\{D_{\alpha_{2}^{\vee}}, \ldots, D_{\alpha_{l}^{\vee}}\right\}\right)$. Let $b \omega_{1}$ be the primitive vector of $\mathbb{R}^{+} \omega_{1}$; we have $1 \leq b \leq l+1$. Suppose first that $\left(v, \alpha_{l-1}\right)=-\delta_{i, 1}$ for each $i<l$; then $v=\sum_{i=1}^{l-1} a_{i} \gamma_{i}-b \omega_{1}$, where the $\gamma_{i}$ are as before. The $a_{i}$ are positive integers, because $-C^{+} \cap \chi_{*}(S) \subset \mathbb{Z}^{+}\left(-\omega_{1}\right)+\sum \mathbb{Z}^{+} \gamma_{i}$; the coefficient of $v$ with respect to $-\omega_{1}$ is $b$ because $-b \omega_{1}$ belongs to the the lattice generated by $\gamma_{1}, \ldots, \gamma_{l-1}$ and $v$. Let $\left\{\widetilde{\lambda}_{1}, \ldots, \widetilde{\lambda}_{l}\right\}$ be the dual basis of $\left\{\gamma_{1}, \ldots, \gamma_{l-1}, v\right\}$; we have $\widetilde{\lambda}_{l}=(1 / b) \lambda_{l}$ and $\widetilde{\lambda}_{i}=\lambda_{i}-\left(a_{i} / b\right) \lambda_{l}$ for each $i<l$. Because $\tilde{X}$ is smooth we have $\widetilde{\omega}_{j}=\frac{1}{l}\left(l \widetilde{\lambda}_{j}-j \widetilde{\lambda}_{l}\right)=\frac{1}{l}\left(l \lambda_{j}-j \lambda_{l}\right)+\frac{1}{l}\left(j-\frac{l a_{j}}{b}-\frac{j}{b}\right) \lambda_{l}$, so $a_{j}=\frac{(b-1) j}{l}$. In particular $l$ divides $b-1$, so either $b=1$ or $b=l+1$. If $b=1$, then the $a_{j}$ are all zero and $\widetilde{X}$ is complete, a contradiction. If $b=l+1$, then $a_{j}=j$ for each $j$ and $v=-\omega_{1}-\omega_{l}$ is a root. Thus $\chi(S)$ must be the weight lattice of $R_{G, \theta}$. Notice that $-\omega_{1}-\omega_{l}=-\alpha_{1}-\ldots-\alpha_{l}$, so $\left\{\alpha_{l-1}, \ldots, \alpha_{1},-\omega_{1}-\omega_{l}\right\}$ is a basis of $\chi_{*}(S)$. Observe that $-\omega_{1}-\omega_{l}$ is fixed by the non trivial automorphism of the Dynkin diagram. Next, suppose that $\left(\alpha_{i}, v\right)=-\delta_{i, l-1}$ for each $i<l$, so $v=-\omega_{l-1}-a \omega_{l}$. We exclude this case because the simple variety associated to cone $\left(\alpha_{2}, \ldots, \alpha_{l}, v\right)$ is not smooth.

We have proved that, if $X$ is not simple, then it is covered by two simple varieties corresponding to the colored cones $\left(\operatorname{cone}\left(\alpha_{1}, \ldots, \alpha_{l-1},-\omega_{1}-\omega_{l}\right),\left\{D_{\alpha_{1}^{\vee}}, \ldots, D_{\alpha_{l-1}^{\vee}}\right\}\right)$ and $\left(\operatorname{cone}\left(\alpha_{2}, \ldots, \alpha_{l},-\omega_{1}-\omega_{l}\right),\left\{D_{\alpha_{2}^{\vee}}, \ldots, D_{\alpha_{l}}\right\}\right)$. If $l$ is at least 3 , then $-\omega_{2}$ does not belongs neither to cone $\left(\alpha_{1}, \ldots, \alpha_{l-1},-\omega_{1}-\omega_{l}\right)$ nor to cone $\left(\alpha_{2}, \ldots, \alpha_{l},-\omega_{1}-\omega_{l}\right)$; thus $l$ must be 2 . In this case, the previous colored cones define a complete variety because cone $\left(-\alpha_{1},-\alpha_{1}-\alpha_{2}\right) \subset \operatorname{cone}\left(\alpha_{2},-\omega_{1}-\omega_{2}\right)$.

4) Suppose that $R_{G, \theta}$ has type $B_{l}$ with $l>2$; then $X$ must be simple. Observe that the dual root system has type $C_{l}$ and $\rho(D(X))=\left\{\alpha_{1}^{\vee}, \ldots, \alpha_{l-1}^{\vee}\right\}$. We can realize $R_{G, \theta}$ as the set $\left\{ \pm\left(e_{i} \pm e_{j}\right) \mid i \neq j\right\} \cup\left\{ \pm e_{i}\right\} \subset \mathbb{R}^{l}$; moreover we can suppose that the basis of $R_{G, \theta}$ is $\left\{e_{1}-e_{2}, \ldots, e_{l-1}-e_{l}, e_{l}\right\}$. The dual root system is $\left\{ \pm\left(e_{i} \pm\right.\right.$ $\left.\left.e_{j}\right) \mid i \neq j\right\} \cup\left\{ \pm 2 e_{i}\right\} \subset \mathbb{R}^{l}$ and has basis $\left\{e_{1}-e_{2}, \ldots, e_{l-1}-e_{l}, 2 e_{l}\right\}$. Notice that the cone generated by $\rho(D(X))$ and $-C^{+}$is equal to the cone cone $\left(\alpha_{1}^{\vee}, \ldots, \alpha_{l-1}^{\vee},-\omega_{1}^{\vee}\right)$. Indeed $-\omega_{2}^{\vee}=\alpha_{1}^{\vee}-2 \omega_{1}^{\vee}$ and $-\omega_{i}^{\vee}=\sum_{j=1}^{i-1} \alpha_{j}^{\vee}-\omega_{1}^{\vee}-\omega_{i-1}^{\vee}$ for $i \geq 3$. If $\chi(S)$ is the root lattice of $R_{G, \theta}$, then $C(X) \cap \chi_{*}(S)$ is generated by $\left\{\alpha_{1}^{\vee}, \ldots, \alpha_{l-1}^{\vee},-\omega_{1}^{\vee}\right\}$ which is a basis of $\chi_{*}(S)$. Now we can proceed as in the case of $A_{l}$ because the Cartan numbers $\left(\alpha_{i}, \alpha_{j}^{\vee}\right)$ with $j<l$ are equal to the ones of $A_{l}$; explicitly we define $f_{i}=$ $-e_{l-i+1}$ and $\gamma_{i}=\alpha_{l-i}$, so that $-\omega_{1}=-\omega_{1}^{\vee}=f_{l}, \gamma_{0}=-f_{1}$ and $\gamma_{i}=\gamma_{i}^{\vee}=f_{i}-f_{i+1}$ 
for each $i=1, \ldots, l-1$. Let $\left\{\lambda_{1}, \ldots, \lambda_{l}\right\}$ be the dual basis of $\left\{\gamma_{1}^{\vee}, \ldots, \gamma_{l-1}^{\vee},-\omega_{1}^{\vee}\right\}$; we have $\lambda_{j}=\sum_{h=1}^{j} f_{h}$, so $(1 / l)\left(l \lambda_{j}-j \lambda_{l}\right)=(1 / l)\left(l \sum_{h=1}^{j} f_{h}-j \sum_{h=1}^{l} f_{h}\right)$ is the $j$-th fundamental weight of the root system generated by $\gamma_{1}, \ldots, \gamma_{l-1}$. We have proved that in this case $X$ is smooth. It is the only possibility because of Lemma 3.4.

$5)$ Suppose now that $R_{G, \theta}$ has type $C_{l}$. Then $X$ must be simple and $\rho(D(X))$ must be equal to $\left\{\alpha_{1}^{\vee}, \ldots, \alpha_{l-1}^{\vee}\right\}$. Observe that the dual root system has type $B_{l}$. We can realize $R_{G, \theta}$ as $\left\{ \pm\left(e_{i} \pm e_{j}\right) \mid i \neq j\right\} \cup\left\{ \pm 2 e_{i}\right\} \subset \mathbb{R}^{l}$ with basis $\left\{e_{1}-e_{2}, \ldots, e_{l-1}-\right.$ $\left.e_{l}, 2 e_{l}\right\}$; the dual root system is $\left\{ \pm\left(e_{i} \pm e_{j}\right) \mid i \neq j\right\} \cup\left\{ \pm e_{i}\right\}$. The cone generated by $\rho(D(X))$ and $-C^{+}$is cone $\left(\alpha_{1}^{\vee}, \ldots, \alpha_{l-1}^{\vee},-\omega_{1}^{\vee}\right)$. Indeed $-\omega_{2}^{\vee}=\alpha_{1}^{\vee}-2 \omega_{1}^{\vee},-\omega_{i}^{\vee}=$ $\sum_{j=1}^{i-1} \alpha_{j}^{\vee}-\omega_{1}^{\vee}-\omega_{i-1}^{\vee}$ for $2<i<l$ and $-2 \omega_{l}^{\vee}=\sum_{j=1}^{l-1} \alpha_{j}^{\vee}-\omega_{1}^{\vee}-\omega_{l-1}^{\vee}$. The lattice $\chi_{*}(S)$ is generated by the restricted coroots because $\omega_{l}^{\vee}$ does not belong to $\mathbb{Z}\left(-\omega_{1}^{\vee}\right) \oplus \bigoplus_{i=1}^{l-1} \mathbb{Z} \alpha_{i}^{\vee}$; thus $G=G^{\theta}$. We have to exclude the cases where $G / H$ is Hermitian, because in such cases $X$ would have rank 2 . The set $\left\{\alpha_{1}^{\vee}, \ldots, \alpha_{l-1}^{\vee},-\omega_{1}^{\vee}\right\}$ is a basis of $\chi_{*}(S)$ because $-\omega_{1}^{\vee}=-\sum_{i=1}^{l} \alpha_{i}^{\vee}$. We define $f_{i}=-e_{l-i+1}$ and $\gamma_{i}=\alpha_{l-i}$, so that $-\omega_{1}=-\omega_{1}^{\vee}=f_{l}, \gamma_{0}=-2 f_{1}$ and $\gamma_{i}=\gamma_{i}^{\vee}=f_{i}-f_{i+1}$ for each $i=1, \ldots, l-1$. Let $\left\{\lambda_{1}, \ldots, \lambda_{l}\right\}$ be the dual basis of $\left\{\gamma_{1}^{\vee}, \ldots, \gamma_{l-1}^{\vee},-\omega_{1}\right\}$, then $\lambda_{j}=$ $\sum_{h=1}^{j} f_{h}$ and $(1 / l)\left(l \lambda_{j}-j \lambda_{l}\right)=(1 / l)\left(l \sum_{i=1}^{j} f_{i}-j \sum_{i=1}^{l} f_{i}\right)$ is the $j$-th fundamental weight of the root system generated by $\gamma_{1}, \ldots, \gamma_{l-1}$. Thus $X$ is smooth if $C(X)=$ $\operatorname{cone}\left(\gamma_{1}^{\vee}, \ldots, \gamma_{l-1}^{\vee},-\omega_{1}\right\}$ and $\chi_{*}(S)=\bigoplus_{i=0}^{l} \mathbb{Z} \gamma_{i}^{\vee}$; it is the only possibility because of Lemma 3.4.

6) Suppose that $R_{G, \theta}$ has type $B_{2}$. We can realize $R_{G, \theta}$ as in the case of $B_{l}$ with $l>2$. First suppose that $\rho(D(\widetilde{X}))=\left\{\alpha_{1}^{\vee}\right\}$ and write $v=-a \omega_{1}^{\vee}-b \omega_{2}^{\vee}$. Let $\left\{\lambda_{1}, \lambda_{2}\right\}$ be the dual basis of $\left\{\alpha_{1}^{\vee}, v\right\}$; we have $\lambda_{1}=-\alpha_{2}-\frac{b}{a+2 b}\left(-\alpha_{1}-2 \alpha_{2}\right)$ and $\lambda_{2}=$ $\frac{1}{a+2 b}\left(-\alpha_{1}-2 \alpha_{2}\right)$. We have $a=1$ because $\frac{1}{2}\left(2 \lambda_{1}-\lambda_{2}\right)=\frac{1}{2}\left(-2 \alpha_{2}-\frac{1+2 b}{a+2 b}\left(-\alpha_{1}-2 \alpha_{2}\right)\right)$ must be $\frac{1}{2} \alpha_{1}$. On the other hand $1+2 b$ divides 2 because $\lambda_{2}$ is a weight, so $b=0$. Hence $v=-\omega_{1}^{\vee}$; in particular $\tilde{X}$ is complete and $\chi(S)$ is the restricted root lattice because $-\omega_{2}^{\vee}=-2 \omega_{1}^{\vee}+\alpha_{1}^{\vee}$. We have proved that there is only one smooth, simple embedding with Picard number one and $\rho(D(\tilde{X}))=\left\{\alpha_{1}^{\vee}\right\}$; moreover it is complete. Thus there is no a smooth, complete, non simple embedding with Picard number one, because otherwise it would strictly contain a simple, open, smooth $G$ subvariety $\widetilde{X}$ with Picard number one and $\rho(D(\widetilde{X}))=\left\{\alpha_{1}^{\vee}\right\}$. Finally, we consider the case in which $X$ is simple and $\rho(D(X))=\left\{\alpha_{2}^{\vee}\right\}$, so $C(X)=\operatorname{cone}\left(-\omega_{2}^{\vee}, \alpha_{2}^{\vee}\right)$. Because of the completeness of $X$, one can study this case exactly as the case 5 ) of $C_{n}$. Observe that if $G / H$ is Hermitian, then we have to consider only the first possibility, because in the second case the Picard number would be 2 .

7) Suppose now that $R_{G, \theta}$ has type $B C_{l}$. We can realize $R_{G, \theta}$ as the set $\left\{ \pm\left(e_{i} \pm\right.\right.$ $\left.\left.e_{j}\right) \mid i \neq j\right\} \cup\left\{ \pm 2 e_{i}\right\} \cup\left\{ \pm e_{i}\right\} \subset \mathbb{R}^{l}$; we can also choose $\left\{e_{1}-e_{2}, \ldots, e_{l-1}-e_{l}, e_{l}\right\}$ as basis of $R_{G, \theta}$. The weight lattice is equal to the root lattice; moreover, $B C_{l}$ coincides with its dual root system in this realization as subset of $\mathbb{R}^{l}$ (but $\frac{2}{(\alpha, \alpha)} \alpha$ can be different from $\alpha$ ). We proceed as in the cases of $B_{l}$ and $C_{l}$. The $G$ variety $X$ must be simple; hence $C(X)$ is the cone generated by $-C^{+}$and $\rho(D(X))$, namely $\left(\operatorname{cone}\left(\alpha_{1}^{\vee}, \ldots, \alpha_{l-1}^{\vee},-\omega_{1}^{\vee}\right)\right.$. Observe that we exclude the case where $l=2$ and $\rho(D(\widetilde{X}))=\left\{\alpha_{2}^{\vee}\right\}$, because $R_{L, \theta}$ would have type $B C_{1}$. The set $\left\{\alpha_{1}^{\vee}, \ldots, \alpha_{l-1}^{\vee},-\omega_{1}^{\vee}\right\}$ is a basis of the restricted coroot lattice because $\omega_{1}^{\vee}=e_{1}$; now, one can prove the smoothness of $X$ exactly as in the case of $B_{n}$.

8) Suppose that $R_{G, \theta}$ has type $D_{l}$. We can realize $R_{G, \theta}$ as the set $\left\{ \pm\left(e_{i} \pm e_{j}\right) \mid i \neq\right.$ j\} $\subset \mathbb{R}^{l}$; moreover we can choose $\left\{e_{1}-e_{2}, \ldots, e_{l-1}-e_{l}, e_{l-1}+e_{l}\right\}$ as basis. In particular, we can we identify the restricted roots with the corresponding coroots. We can suppose, up to an automorphism of the Dynkin diagram, that $\rho(D(\widetilde{X}))=$ $\left\{\alpha_{1}, \ldots, \alpha_{l-1}\right\}$. Suppose first that $\left(\alpha_{i}, v\right)=-\delta_{i, 1}$ for each $i<l$, so $v=\sum_{i=1}^{l-2}(i a+i-$ 
1) $\alpha_{i}+\frac{(l-2) a+l-3}{2} \alpha_{l-1}+\frac{l a+l-1}{2} \alpha_{l}$ for an appropriate constant $a$. The restricted root $\alpha_{l}$ belongs to $\mathbb{Z} v \oplus \bigoplus_{i=1}^{l-1} \mathbb{Z} \alpha_{i}$, thus its coordinate with respect to $v$, namely $\frac{2}{l a+l-1}$, is an integer. On the other hand $v=-\omega_{1}-b \omega_{l}$; we obtain $2 a=-b-2$ comparing the coordinates of $v$ in the bases of the simple restricted roots, respectively of the fundamental spherical weights. The previous facts imply that $b=0$, so $v=$ $-\omega_{1}$. We set $f_{i}=-e_{l-i+1}$ and $\gamma_{i}=\alpha_{l-i}$, so that $-\omega_{1}=f_{l}, \gamma_{0}=-f_{1}-f_{2}$ and $\gamma_{i}=f_{i}-f_{i+1}$ for each $i=1, \ldots, l-1$. Defining $\left\{\lambda_{1}, \ldots, \lambda_{l}\right\}$ as the dual basis of $\left\{\gamma_{1}, \ldots, \gamma_{l-1}, v\right\}$, we have $\lambda_{j}=\sum_{i=1}^{j} f_{i}$ for each $j$, thus $\frac{1}{l}\left(l \lambda_{j}-j \lambda_{l}\right)$ is the $j$ th fundamental weight of $R_{L, \theta}$. Moreover the lattice $\chi_{*}(S)$ is freely generated by $\omega_{1}, \ldots, \omega_{l-2}, \omega_{l-1}+\omega_{l}, 2 \omega_{l}$.

Suppose now that $\left(\alpha_{i}, v\right)=-\delta_{i, l-1}$ for each $i<l$, thus $v=-\omega_{l-1}-b \omega_{l}$. Moreover $v$ has to be equal to $a \sum_{i=1}^{l-2} i \alpha_{i}+\frac{(l-2) a-1}{2} \alpha_{l-1}+\frac{l a+1}{2} \alpha_{l}$ for an appropriate constant $a$. We compare the coordinates of $v$ in the bases of the simple restricted roots, respectively of the fundamental spherical weights, obtaining $2 a=-b-1$. On the other hand, the restricted root $\alpha_{l}$ must belong to the lattice generated by $\left\{\alpha_{1}, \ldots, \alpha_{l-1}, v\right\}$, so its coordinate with respect to $v$, namely $\frac{2}{l a+1}$, must be an integer; hence $a=-\frac{1}{2}, v=-\omega_{l-1}$ and $l$ is equal either to 4 or to 6 . If $l=4$ we can reduce this case to the one where $C(\tilde{X})=\operatorname{cone}\left(\alpha_{1}, \alpha_{2}, \alpha_{3},-\omega_{1}\right)$ by an automorphism of the Dynkin diagram. Finally consider the case where $l=6$ and $C(\widetilde{X})=$ cone $\left(\alpha_{1}, \ldots, \alpha_{5},-\omega_{5}\right)$. The variety $\widetilde{X}$ must be complete because the simple symmetric variety corresponding to $\left(\right.$ cone $\left.\left(\alpha_{1}, \ldots, \alpha_{4}, \alpha_{6},-\omega_{5}\right),\left\{D_{\alpha_{1}^{\vee}}, \ldots, D_{\alpha_{4}^{\vee}}, D_{\alpha_{6}^{\vee}}\right\}\right)$ is not smooth. But $-\omega_{3}$ is equal to $-\frac{3}{2} \omega_{5}-\frac{1}{4} \alpha_{1}-\frac{1}{2} \alpha_{2}-\frac{3}{4} \alpha_{3}+\frac{3}{4} \alpha_{5}$, so it does not belong to cone $\left(\alpha_{1}, \ldots, \alpha_{5},-\omega_{5}\right)$, a contradiction.

We have proved that, if $l$ is different from 4 , then there is at most one complete symmetric variety with the requested properties and it is such that: 1) $\chi_{*}(S)=$ $\left.\bigoplus_{i=1}^{l-2} \mathbb{Z} \omega_{i} \oplus \mathbb{Z}\left(\omega_{l-1}+\omega_{l}\right) \oplus \mathbb{Z} 2 \omega_{l} ; 2\right)$ the corresponding colored fan is formed by $\left(\operatorname{cone}\left(\alpha_{1}, \ldots, \alpha_{l-1},-\omega_{1}\right),\left\{D_{\alpha_{1}^{\vee}}, \ldots, D_{\alpha_{l-1}^{\vee}}\right\}\right)$, by $\left(\operatorname{cone}\left(\alpha_{1}, \ldots, \alpha_{l-2}, \alpha_{l},-\omega_{1}\right),\left\{D_{\alpha_{1}^{\vee}}, \ldots\right.\right.$, $\left.\left.D_{\alpha_{l-2}^{\vee}}, D_{\alpha_{l}^{\vee}}\right\}\right)$ and by their colored faces. We have to show that these combinatorial data define a variety and that this variety is complete. To verify that these colored cones define a colored fan it is sufficient to prove that the intersection of cone $\left(\alpha_{1}, \ldots, \alpha_{l-1},-\omega_{1}\right)$ with cone $\left(\alpha_{1}, \ldots, \alpha_{l-2}, \alpha_{l},-\omega_{1}\right)$ is cone $\left(\alpha_{1}, \ldots, \alpha_{l-2},-\omega_{1}\right)$. Let $\sum_{i=1}^{l-2} a_{i} \alpha_{i}+b \alpha_{l}-c \omega_{1}=\sum_{i=1}^{l-2}\left(a_{i}-2 b\right) \alpha_{i}-b \alpha_{l-1}+(-c+2 b) \omega_{1}$ be a vector in the intersection, then $b$ is equal to 0 . The variety $X$ is complete because cone $\left(\alpha_{1}, \ldots, \alpha_{l-1},-\omega_{1}\right) \cap-C^{+}=\operatorname{cone}\left(-\omega_{1}, \ldots,-\omega_{l-2},-\omega_{l-1}-\omega_{l},-\omega_{l}\right)$ and cone $\left(\alpha_{1}, \ldots, \alpha_{l-2}, \alpha_{l},-\omega_{1}\right) \cap-C^{+}=\operatorname{cone}\left(-\omega_{1}, \ldots,-\omega_{l-2},-\omega_{l-1}-\omega_{l},-\omega_{l-1}\right)$. If $l$ is equal to 4 , we can proceed in an analogous way.

If $R_{G, \theta}$ has type $E_{l}$, we number the simple roots so that $\alpha_{1}, \widehat{\alpha}_{2}, \alpha_{3}, \ldots, \alpha_{l}$ generated a root system of type $A_{l-1}$; moreover we choose the inner product so that $\alpha_{i}=\alpha_{i}^{\vee}$ for each $i$. Thus $\rho(D(\widetilde{X}))$ has to be $\left\{\alpha_{1}, \widehat{\alpha}_{2}, \alpha_{3}, \ldots, \alpha_{l}\right\}$ and $\widetilde{X}$ must be complete.

9) Suppose that $R_{G, \theta}$ has type $E_{6}$. Up to an automorphism of the Dynkin diagram, we can suppose $\left(\alpha_{i}, v\right)=-\delta_{i, 6}$ for each $i$ different from 2 . Thus $v=$ $\sum a_{i} \alpha_{i}=-a \omega_{2}-\omega_{6}$, where $a$ is a positive integer. We compare the coordinates of $v$ in the bases of the simple roots, respectively of the fundamental weights, obtaining $a_{2}=-2 a-1$. On the other hand, $\alpha_{2}$ belongs to the lattice generated by $\alpha_{1}, \alpha_{3}, \ldots, \alpha_{6}, v$, thus $\left(a_{2}\right)^{-1}$ is an integer; hence $v$ has to be $-\omega_{6}$. The weight $-3 \omega_{1}$ is equal to $-3 \omega_{6}-2 \alpha_{1}-\alpha_{3}+\alpha_{5}+2 \alpha_{6}$, so it does not belong to $C(\tilde{X})$. Thus $\tilde{X}$ is not complete, a contradiction.

10) Suppose that $R_{G, \theta}$ has type $E_{7}$ and write $v=\sum a_{i} \alpha_{i}$. The coefficients $a_{i}$ belong to $\frac{1}{2} \mathbb{Z}$ because $v$ is a weight. Moreover $\left(a_{2}\right)^{-1}$ is an integer, because $\alpha_{2}$ belongs to the lattice generated by $\alpha_{1}, \alpha_{3}, \ldots, \alpha_{7}, v$, thus $a_{2}$ is equal either to 
\pm 1 or to $\pm \frac{1}{2}$. First suppose that $\left(\alpha_{i}, v\right)=-\delta_{i, 1}$ for each $i$ different from 2 , so $v=-\omega_{1}-a \omega_{2}$ for an appropriate positive integer $a$. We compare the coordinates of $v$ in the bases of the simple restricted roots, respectively of the fundamental spherical weights, obtaining $a=\frac{2}{7}\left(-a_{2}-2\right)$; thus $a$ is not integer, a contradiction. Finally, suppose that $\left(\alpha_{7}, v\right)=-1$. We have only to study the basis $\left\{\lambda_{1}, \ldots, \lambda_{7}\right\}$, where $\lambda_{1}=\omega_{1}-\frac{a_{1}}{a_{2}} \omega_{2}, \lambda_{i}=\omega_{i+1}-\frac{a_{i+1}}{a_{2}} \omega_{2}$ for $1<i<7$ and $\lambda_{7}=\frac{1}{a_{2}} \omega_{2}$. Comparing the second coordinate of $\frac{1}{7}\left(7 \lambda_{1}-\lambda_{7}\right)$ (with respect to the basis $\left\{\alpha_{1}, \ldots, \alpha_{7}\right\}$ ) with the one of the first fundamental weight of $R_{L, \theta}$, we obtain $7 a_{1}=4 a_{2}-1$. Thus $2\left(4 a_{2}-1\right)$ is integral multiple of 7 , a contradiction.

11) Suppose that $R_{G, \theta}$ has type $E_{8}$ and write $v=\sum a_{i} \alpha_{i}$. Notice that the root lattice of $R_{G, \theta}$ is equal to the weight lattice. The integer $a_{2}$ is \pm 1 because $\left\{\alpha_{1}, \alpha_{3}, \ldots, \alpha_{8}, v\right\}$ is a basis of $\chi_{*}(S)$. First, suppose that $v=-\omega_{1}-a \omega_{2}$ for an appropriate positive integer $a$. We compare the coordinates of $v$ in the two bases, respectively of simple restricted roots and fundamental spherical weights, obtaining $a=\frac{1}{8}\left(-a_{2}-5\right)$; so $a$ is not integer, a contradiction. Finally, suppose that $\left(\alpha_{8}, v\right)=$ -1 . We have only to study the indexed basis $\left\{\lambda_{1}, \ldots, \lambda_{8}\right\}$ with $\lambda_{1}=\omega_{1}-\frac{a_{1}}{a_{2}} \omega_{2}$, $\lambda_{i}=\omega_{i+1}-\frac{a_{i+1}}{a_{2}} \omega_{2}$ for $1<i<8$ and $\lambda_{8}=\frac{1}{a_{2}} \omega_{2}$. Comparing the second coordinate of $\frac{1}{8}\left(8 \lambda_{1}-\lambda_{8}\right)$ (with respect to the basis $\left\{\alpha_{1}, \ldots, \alpha_{8}\right\}$ ) with the one of the first fundamental weight of $R_{L, \theta}$, we obtain $8 a_{1}=5 a_{2}-1$. On the other hand, $5 a_{2}-1$ cannot be a integral multiple of 8 , a contradiction.

12) If $R_{G, \theta}$ has type $F_{4}$, then $R_{L, \theta}$ cannot have type $A_{3}$ and there are no simple smooth varieties with Picard number one.

13) Suppose that $R_{G, \theta}$ has type $G_{2}$ (and assume $\alpha_{1}$ short). Write $v=-a \omega_{1}^{\vee}-$ $b \omega_{2}^{\vee}$ and observe that the weight lattice coincides with the root lattice. First, suppose that $D(X)$ contains $D_{\alpha_{1}^{\vee}}$, hence $X$ contains a simple smooth subvariety $\widetilde{X}$ with $C(\tilde{X})=\operatorname{cone}\left(\alpha_{1}^{\vee}, v\right)$. Let $\left\{\lambda_{1}, \lambda_{2}\right\}$ be the dual basis of $\left\{\alpha_{1}^{\vee}, v\right\}$. We have $\lambda_{1}=-\frac{1}{3} \alpha_{2}+\frac{b}{9 a+6 b}\left(3 \alpha_{1}+2 \alpha_{2}\right)$ and $\lambda_{2}=-\frac{1}{3 a+2 b}\left(3 \alpha_{1}+2 \alpha_{2}\right)$. Thus $3 a+2 b$ must divide 2 and 3 , so it must be 1 , a contradiction. Thus $X$ is simple and $C(X)=$ $\operatorname{cone}\left(\alpha_{2}^{\vee},-\omega_{2}^{\vee}\right)$. The dual basis of $\left\{\alpha_{2}^{\vee},-\omega_{2}^{\vee}\right\}$ is $\left\{-\alpha_{1},-\alpha_{2}-2 \alpha_{1}\right\}$. Moreover $\frac{1}{2}\left(2 \lambda_{1}-\lambda_{2}\right)=\frac{1}{2} \alpha_{2}$ is the fundamental weight of $R_{L, \theta}$. Thus the variety associated to $\left(\operatorname{cone}\left(\alpha_{2}^{\vee},-\omega_{2}^{\vee}\right), D_{\alpha_{2}^{\vee}}\right)$ is smooth.

Lemma 3.5. Every smooth completion $X$ of $G / H$ with Picard number 1 is projective.

Proof. It is sufficient to consider the varieties which are not simple. There are exactly two maximal colored cones, say $\left(\varsigma_{1}, I_{1}\right)$ and $\left(\varsigma_{2}, I_{2}\right)$; moreover there are exactly two colors, say $D_{1} \in I_{1}$ and $D_{2} \in I_{2}$, which does not belong to $I_{1} \cap I_{2}$. We claim that $D_{1}+D_{2}$ is an ample divisor. Indeed, let $\varphi$ be the function over $\varsigma_{1} \cup \varsigma_{2}$ corresponding to $D_{1}+D_{2}$ (see Theorem 3.1 in [1]) and let $l_{i}$ be the linear function which coincides with $\varphi$ over $\varsigma_{i}$. The cone $\varsigma_{i}$ is generated by $\rho\left(D_{i}\right)$ and $\varsigma_{1} \cap \varsigma_{2} ;$ moreover $\rho\left(D_{1}\right)$ and $\rho\left(D_{2}\right)$ are permutated by the reflection with respect to the hyperplane generated by $\varsigma_{1} \cap \varsigma_{2}$. Thus $l_{1}\left(\rho\left(D_{2}\right)\right)=-1<1=\varphi\left(\rho\left(D_{2}\right)\right)$ and $l_{2}\left(\rho\left(D_{1}\right)\right)=-1<1=\varphi\left(\rho\left(D_{1}\right)\right)$, because $\operatorname{span}_{\mathbb{R}}\left(\varsigma_{1} \cap \varsigma_{2}\right)$ is the kernel of both $l_{1}$ and $l_{2}$. Hence $\varphi$ is strictly convex over the colored fan of $X$. Moreover $D(X)=D(G / H)$, therefore $D_{1}+D_{2}$ is ample by Theorem 3.3 in [1].

We have proved the following theorem:

Theorem 3.1. Let $G$ be a semisimple simply connected group and let $G / H$ be a homogeneous symmetric variety. Suppose that there is a smooth, complete embedding $X$ of $G / H$ with Picard number one. Then:

- Given $G / H$, there is, up to equivariant isomorphism, at most one embedding with the previous properties. 
- The symmetric variety $X$ is projective.

- The number of colors of $G / H$ is equal to the rank $l$ of $G / H$; in particular there are no exceptional roots. If, in addition, $l$ is at least 3 , then $G / H$ is not Hermitian.

- The restricted root system $R_{G, \theta}$ is irreducible or has type $A_{1} \times A_{1}$.

- We have two possibilities:

(1) $X$ is simple and $D(X)$ has cardinality $l-1$.

(2) $X$ contains two closed orbits, $D(X)$ is equal to $D(G / H)$ and $H$ has index two in $N_{G}\left(G^{\theta}\right)$.

In particular, $X$ is simple if $H=N_{G}\left(G^{\theta}\right)$.

- We have the following classification depending on the type of the restricted root system $R_{G, \theta}$ :

(1) If $R_{G, \theta}$ has type $A_{1} \times A_{1}$, then $\chi(S)$ has basis $\left\{2 \omega_{1}, \omega_{1}+\omega_{2}\right\}$; in particular $H$ has index two in $N_{G}\left(G^{\theta}\right)$. Moreover, $X$ has two closed orbits; the maximal colored cones of the colored fan of $X$ are $\left(\right.$ cone $\left(\alpha_{1}^{\vee},-\omega_{1}^{\vee}-\omega_{2}^{\vee}\right)$, $\left.\left\{D_{\alpha_{1}^{\vee}}\right\}\right)$ and (cone $\left.\left(\alpha_{2}^{\vee},-\omega_{1}^{\vee}-\omega_{2}^{\vee}\right),\left\{D_{\alpha_{2}^{\vee}}\right\}\right)$.

(2) If $l=1$, then $G / H$ can be isomorphic neither to $S L_{n+1} / S\left(L_{1} \times L_{n}\right)$, nor to $\mathrm{SL}_{2} / \mathrm{SO}_{2}$. With such hypothesis, $\mathrm{G} / \mathrm{H}$ has a unique non trivial embedding which is simple, projective, smooth and with Picard number 1.

(3) If $R_{G, \theta}$ has type $A_{l}$ with $l>1$, we have the following possibilities:

- $H=N_{G}\left(G^{\theta}\right)$ and $X$ is simple. In this case $X$ is associated either to the colored cone $\left(\right.$ cone $\left.\left(\alpha_{1}^{\vee}, \ldots, \alpha_{l-1}^{\vee},-\omega_{1}^{\vee}\right),\left\{D_{\alpha_{1}^{\vee}}, \ldots, D_{\alpha_{l-1}^{\vee}}\right\}\right)$ or to the one (cone $\left.\left(\alpha_{2}^{\vee}, \ldots, \alpha_{l}^{\vee},-\omega_{l}^{\vee}\right),\left\{D_{\alpha_{2}^{\vee}}, \ldots, D_{\alpha_{l}^{\vee}}\right\}\right)$;

- $H=G^{\theta}$ and $l=2$. In this case $X$ has two closed orbits. The maximal colored cones of the colored fan of $X$ are $\left(\right.$ cone $\left(\alpha_{1}^{\vee},-\omega_{1}^{\vee}-\right.$ $\left.\left.\omega_{2}^{\vee}\right),\left\{D_{\alpha_{1}^{\vee}}\right\}\right)$ and $\left(\operatorname{cone}\left(\alpha_{2}^{\vee},-\omega_{1}^{\vee}-\omega_{2}^{\vee}\right),\left\{D_{\alpha_{2}^{\vee}}\right\}\right)$.

(4) If $R_{G, \theta}$ has type $B_{2}$, then $X$ is simple and we have the following possibilities:

- $H=N_{G}\left(G^{\theta}\right)$ and $X$ is associated to the colored cone (cone $\left(\alpha_{1}^{\vee}\right.$, $\left.\left.-\omega_{1}^{\vee}\right),\left\{D_{\alpha_{1}^{\vee}}\right\}\right)$;

- $H=G^{\theta}$ and $X$ is associated to the colored cone $\left(\right.$ cone $\left(\alpha_{2}^{\vee},-\omega_{2}^{\vee}\right)$, $\left.\left\{D_{\alpha_{2}}\right\}\right)$. Moreover $G / H$ cannot be Hermitian.

(5) If $R_{G, \theta}$ has type $B_{l}$ with $l>2$, then $H=N_{G}\left(G^{\theta}\right)$, $X$ is simple and is associated to the colored cone $\left(\right.$ cone $\left.\left(\alpha_{1}^{\vee}, \ldots, \alpha_{l-1}^{\vee},-\omega_{1}^{\vee}\right),\left\{D_{\alpha_{1}^{\vee}}, \ldots, D_{\alpha_{l-1}^{\vee}}\right\}\right)$.

(6) If $R_{G, \theta}$ has type $C_{l}$, then $H=G^{\theta}, X$ is simple and corresponds to the colored cone (cone $\left.\left(\alpha_{1}^{\vee}, \ldots, \alpha_{l-1}^{\vee},-\omega_{1}^{\vee}\right),\left\{D_{\alpha_{1}^{\vee}}, \ldots, D_{\alpha_{l-1}^{\vee}}\right\}\right)$. Moreover $G / H$ cannot be Hermitian.

(7) If $R_{G, \theta}$ has type $B C_{l}$ with $l>1$, then $H=N_{G}\left(G^{\theta}\right)=G^{\theta}, X$ is simple and corresponds to the colored cone $\left(\right.$ cone $\left(\alpha_{1}^{\vee}, \ldots, \alpha_{l-1}^{\vee},-\omega_{1}^{\vee}\right),\left\{D_{\alpha_{1}^{\vee}}, \ldots\right.$, $\left.\left.D_{\alpha_{l-1}^{\vee}}\right\}\right)$.

(8) If $R_{G, \theta}$ has type $D_{l}$ with $l>4$, then $\chi_{*}(S)$ is freely generated by $\omega_{1}^{\vee}, \ldots, \omega_{l-2}^{\vee}, \omega_{l-1}^{\vee}+\omega_{l}^{\vee}, 2 \omega_{l}^{\vee}$; in particular $H$ has index two in $N_{G}\left(G^{\theta}\right)$. $X$ has two closed orbits; the maximal colored cones of the colored fan of $X$ are $\left(\right.$ cone $\left.\left(\alpha_{1}^{\vee}, \ldots, \alpha_{l-1}^{\vee},-\omega_{1}^{\vee}\right),\left\{D_{\alpha_{1}^{\vee}}, \ldots, D_{\alpha_{l-1}^{\vee}}\right\}\right)$ and $\left(\operatorname{cone}\left(\alpha_{1}^{\vee}, \ldots\right.\right.$, $\left.\left.\alpha_{l-2}^{\vee}, \alpha_{l}^{\vee},-\omega_{1}^{\vee}\right),\left\{D_{\alpha_{1}^{\vee}}, \ldots, D_{\alpha_{l-2}^{\vee}}, D_{\alpha_{l}^{\vee}}\right\}\right)$.

(9) If $R_{G, \theta}$ has type $D_{4}$, then $H$ has index two in $N_{G}\left(G^{\theta}\right)$ and $X$ has two closed orbits. If $\chi_{*}(S)=\mathbb{Z} \omega_{i}^{\vee} \oplus \mathbb{Z} \omega_{2}^{\vee} \oplus \mathbb{Z}\left(\omega_{j}^{\vee}+\omega_{k}^{\vee}\right) \oplus \mathbb{Z} 2 \omega_{k}^{\vee}$, then the maximal colored cones of the colored fan of $X$ are $\left(\operatorname{cone}\left(\alpha_{i}^{\vee}, \alpha_{2}^{\vee}\right.\right.$, $\left.\left.\alpha_{j}^{\vee},-\omega_{i}^{\vee}\right),\left\{D_{\alpha_{i}^{\vee}}, D_{\alpha_{2}^{\vee}}, D_{\alpha_{j}^{\vee}}\right\}\right)$ and $\left(\operatorname{cone}\left(\alpha_{i}^{\vee}, \alpha_{2}^{\vee}, \alpha_{k}^{\vee},-\omega_{i}^{\vee}\right),\left\{D_{\alpha_{i}^{\vee}}, D_{\alpha_{2}^{\vee}}\right.\right.$, $\left.D_{\alpha_{k}^{\vee}}\right\}$ ). 
(10) The type of $R_{G, \theta}$ cannot be $E_{6}, E_{7}, E_{8}$ or $F_{4}$.

(11) If $R_{G, \theta}$ has type $G_{2}$ then $H=N_{G}\left(G^{\theta}\right)=G^{\theta}, X$ is simple and is associated to the colored cone (cone $\left.\left(\alpha_{2}^{\vee},,-\omega_{2}^{\vee}\right),\left\{D_{\alpha_{2}^{\vee}}\right\}\right)$.

\section{ACKNOWLEDGMENTS}

We would like to thank M. Brion for many helpful suggestions.

\section{ERrata CORRIGE TO "GEOMETRICAL DESCRIPTION OF SMOOTH PROJECTIVE SYMMETRIC VARIETIES WITH PICARD NUMBER EQUAL TO ONE"}

In Theorem 2 of [13] it is said that "the smooth completion $X$ of $G_{2} /\left(S L_{2} \times S L_{2}\right)$ with $\operatorname{Pic}(X)=\mathbb{Z}$ parametrizes the subspaces $W$ of $\mathbb{C}^{7}$ such that $W \oplus \mathbb{C} 1$ is a subalgebra of $\mathbb{O}_{\mathbb{C}}$ isomorphic to the complexified quaternions." I would to thank $\mathrm{P}$. Chaput which pointed to me that I have only proved that $X$ parametrizes the subspace such that $W \oplus \mathbb{C} 1$ is an associative subalgebra of $\mathbb{O}_{\mathbb{C}}$ of dimension four. But $W \oplus \mathbb{C} 1$ it is not always a composition algebra. Indeed, while $W \oplus \mathbb{C} 1$ is a composition algebra if $W$ is in the open $G_{2}$-orbit, when $W$ is in the closed $G_{2}$-orbit, $W \oplus \mathbb{C} 1$ is isomorphic to the exterior algebra $\wedge^{*} \mathbb{C}^{2}$ of $\mathbb{C}^{2}$. In particular, the proof of Proposition 1, $\S 2.2$ of [13] holds if one deletes the last line.

\section{REFERENCES}

[1] M. BRION, Groupe de Picard et nombres caractéristiques des variétés sphériques, Duke Math. J. 58, (1989), no. 2, 397-424.

[2] M. BRION, Sur la géométrie des variétés sphériques, Comm. Math. Helv., 66, (1991), no. 2, 237-262.

[3] M. BRION, Variétés sphériques, Notes de cours, 1997.

[4] R. CHIRIVÌ and A. MAFFEI, The ring of sections of a complete symmetric variety, J. Algebra, 261, (2003), no. 2, 310-326.

[5] C. De CONCINI and C. PROCESI, Complete symmetric varieties, in Invariant Theory (Montecatini 1982), Lecture Notes in Math, 996, 1-44, Springer-Verlag, New York, 1983.

[6] C. De CONCINI and T. A. SPRINGER, Compactification of symmetric varieties. Dedicated to the memory of Claude Chevalley, Transform. Groups, 4 (1999), no. 2-3, 273-300.

[7] W. FULTON, J. HARRIS, Representation theory. A first course, Graduate Texts in Mathematics, 129, Springer-Verlag (1991).

[8] J. E, HUMPHREYS, Introduction to Lie algebras and representation theory, Graduate Texts in Mathematics, 9, Springer-Verlag (1972).

[9] J. M. LANDSBERG and L. MANIVEL, The projective geometry of Freudenthal's magic square, Journal of Algebra, 239 (2001), no. 2, 477-512.

[10] D. LUNA, Slices étales, Mém. Soc. Math. Fr. (N.S.), 33 (1973), 81-105.

[11] B. PASQUIER, On some smooth projective two-orbit varieties with Picard number 1, Math. Ann. 344 (2009), no. 4, 963987.

[12] L. E. RENNER, Classification of semisimple algebraic monoids, Trans. Amer. Math. Soc. 292 (1985), no. 1, 193-223.

[13] A. RUZZI, Geometrical description of smooth projective symmetric varieties with Picard number one, Transform. groups 15 (2010), no. 1, 201-226.

[14] A. RUZZI, Fano symmetric varieties with low rank, preprint, arXiv : math.AG/1012.4171

[15] R. STEINBERG, Endomorphisms of linear algebraic groups, Memories of the AMS, 80 (1980).

[16] D. A. TIMASHEV, Equivariant compactifications of reductive groups, Sbornik. Mathematics, 194 (2003), no. 3-4, 589-616.

[17] D. A. TIMASHEV, Homogeneous spaces and equivariant embeddings, preprint, arXiv: math.AG/0602228.

[18] T. VUST, Plongements d'espaces symétriques algébriques: une classification, Ann. Scuola Norm. Pisa Cl. Sci. (4), 17, (1990), n. 2, 165-195. 